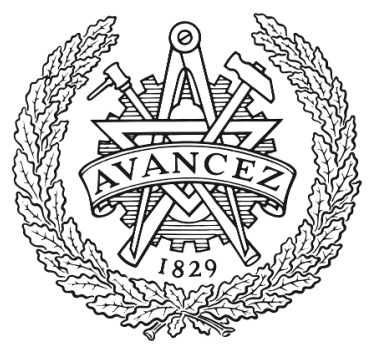

CHALMERS

UNIVERSITY OF TECHNOLOGY

\title{
Zooming in to Massive Star Birth
}

Downloaded from: https://research.chalmers.se, 2023-04-26 05:21 UTC

Citation for the original published paper (version of record):

Kong, S., Tan, J., Caselli, P. et al (2018). Zooming in to Massive Star Birth. Astrophysical Journal, 867(2). http://dx.doi.org/10.3847/1538-4357/aae1b2

N.B. When citing this work, cite the original published paper. 


\title{
Zooming in to Massive Star Birth
}

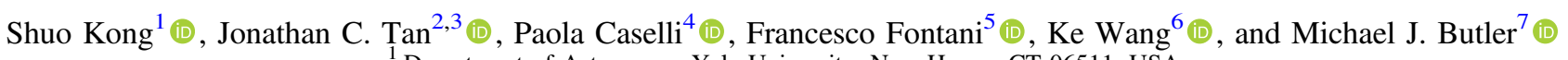 \\ ${ }^{1}$ Department of Astronomy, Yale University, New Haven, CT 06511, USA \\ ${ }^{2}$ Department of Space, Earth and Environment, Chalmers University of Technology, Gothenburg, Sweden \\ ${ }^{3}$ Department of Astronomy, University of Virginia, Charlottesville, VA 22904, USA \\ ${ }^{4}$ Max-Planck-Institute for Extraterrestrial Physics (MPE), Giessenbachstr. 1, D-85748 Garching, Germany \\ ${ }^{5}$ INAF-Osservatorio Astrofisico di Arcetri, L.go E. Fermi 5, I-50125 Florence, Italy \\ ${ }^{6}$ European Southern Observatory (ESO), Karl-Schwarzschild-Str. 2, D-85748 Garching, Germany \\ ${ }^{7}$ Max Planck Institute for Astronomy, Königstuhl 17, D-69117 Heidelberg, Germany \\ Received 2017 January 20; revised 2018 July 24; accepted 2018 August 15; published 2018 November 2
}

\begin{abstract}
We present high-resolution ( 0 !'2,1000 au) $1.3 \mathrm{~mm}$ ALMA observations of the massive infrared dark cloud clump, G028.37+00.07-C1, thought to harbor the early stages of massive star formation. Using $\mathrm{N}_{2} \mathrm{D}^{+}(3-2)$, we resolve the previously identified C1-S core, separating the bulk of its emission from two nearby protostellar sources. C1-S is thus identified as a massive $\left(\sim 50 M_{\odot}\right)$, compact $(\sim 0.1$ pc diameter $)$ starless core, e.g., with no signs of outflow activity. Being highly deuterated, this is a promising candidate for a pre-stellar core on the verge of collapse. An analysis of its dynamical state indicates a sub-virial velocity dispersion compared to a trans-Alfvénic turbulent core model. However, virial equilibrium could be achieved with sub-Alfvénic conditions involving magnetic field strengths of $\sim 2 \mathrm{mG}$.
\end{abstract}

Key words: ISM: jets and outflows - ISM: kinematics and dynamics - ISM: magnetic fields - stars: formation stars: massive - stars: protostars

Supporting material: machine-readable table

\section{Introduction}

One way to distinguish between different theoretical models of massive star formation is to study the initial conditions of gas before formation of a massive protostar (see, e.g., Tan et al. 2014 for a review). Core accretion models, e.g., the turbulent core model of McKee \& Tan (2002, 2003, hereafter MT03), assume that this initial condition is a massive pre-stellar core (PSC). By analogy with low-mass PSCs (see, e.g., Caselli \& Ceccarelli 2012; Friesen et al. 2014), these cores are expected to be highly deuterated and thus well traced by species such as $\mathrm{N}_{2} \mathrm{D}^{+}$. On the other hand, competitive accretion models (e.g., Bonnell et al. 2001; Wang et al. 2010) do not involve massive PSCs as the initial conditions for massive star formation.

Tan et al. (2013, hereafter T13) used $\mathrm{N}_{2} \mathrm{D}^{+}(3-2)$ to identify the C1-S core in a pilot survey with ALMA in Cycle 0 at 2!!3 resolution of four infrared dark cloud (IRDC) clumps (see also Kong et al. 2017 for an extension of this survey to 32 more IRDC clumps). The C1-S core is at the western end of the massive IRDC G028.37+00.07 at a distance of about $5 \mathrm{kpc}$ (Rathborne et al. 2006). Kong et al. (2016) measured the deuteration fraction, $D_{\mathrm{frac}}^{\mathrm{N} 2 \mathrm{H}+} \equiv\left[\mathrm{N}_{2} \mathrm{D}^{+}\right] /\left[\mathrm{N}_{2} \mathrm{H}^{+}\right]$, in this "core" (i.e., roughly $3 . " 5$ in radius) to be $\simeq 0.2$ to 0.7 , which, by comparison with the models of Kong et al. (2015), may indicate a relatively old astrochemical age compared to the local free-fall time of the core.

Tan et al. (2016, hereafter T16) presented results from the compact configuration (i.e., 1".5 resolution) follow-up of the C1-S region with ALMA in Cycle 2, in particular reporting the detection of two protostellar outflow sources in the vicinity (i.e., within $\sim 0.1 \mathrm{pc}$ ) of the core center (see also Feng et al. 2016). If these protostars are embedded in the core, then this would obviously invalidate its status as a PSC. Such a situation arose for the massive IR-quiet core N63 (Bontemps et al. 2010), which was later observed to host a powerful, collimated outflow (Duarte-Cabral et al. 2013). The only other previously reported candidate massive PSC is G11.920.61MM2, detected solely by its continuum emission (Cyganowski et al. 2014). However, the non-detection of any molecular lines toward this source is peculiar and makes it difficult to assess the reliability of the structure, e.g., via a dynamical mass measurement.

Here we present the full results of our Cycle 2 observations of C1-S, combining observations in compact and extended configurations that achieve 0 ". 2 resolution. With these higherresolution data, we are able to spatially and kinematically resolve the $\mathrm{N}_{2} \mathrm{D}^{+}(3-2)$ core from the nearby protostars, so we conclude that it remains a promising candidate to be a massive PSC.

\section{Observations}

\subsection{ALMA Observations}

The observations were carried out during ALMA Cycle 2, under the project 2013.1.00248.S (PI: Tan) using two configurations of the $12 \mathrm{~m}$ array. The observation in the compact configuration (C34-1) was performed on 2015 April 5 (UTC) with $3412 \mathrm{~m}$ antennas and a total on-source integration time of $2087 \mathrm{~s}$. The baselines range from $15 \mathrm{~m}$ to $327 \mathrm{~m}$, corresponding to angular scales from $10^{\prime \prime}$ to $1^{\prime \prime}$. The angular resolution obtained from this observation is about 1 " 5 . The bandpass calibrator was J1924-2914; the flux calibrator was Neptune; the gain calibrator was J1912-0804; and the delay calibrator was J1902-0458. The system temperature was about $85 \mathrm{~K}$. The data in the extended configuration (C34-6) were taken on 2015 July 3 (UTC) with $4312 \mathrm{~m}$ antennas and a total on-source integration time of $4174 \mathrm{~s}$. The baselines range from $34 \mathrm{~m}$ to $1574 \mathrm{~m}$, corresponding to angular scales from 2 "! 3 to 0 !! 2 . The 
angular resolution obtained from this observation is about 0 ". 25 . The bandpass calibrator was $\mathrm{J} 1751+0939$; the flux calibrator was Titan; the gain calibrator was J1827-0405; and the delay calibrator was J1912-0804. The system temperature was about $95 \mathrm{~K}$. Both observations targeted R.A. $=18^{\mathrm{h}} 42^{\mathrm{m}} 46^{\mathrm{s}} .585634$, decl. $=-04^{\circ} 04^{\prime} 12^{\prime \prime} 36111(\mathrm{~J} 2000)$, which is in between $\mathrm{C} 1-\mathrm{N}$ and $\mathrm{C} 1-\mathrm{S}$ (see T16). The primary beam FWHM was $\sim 26^{\prime \prime}$, covering both sources.

The observations were carried out in ALMA band 6. Four basebands and seven spectral windows were used. Baseband 1 was set to a single spectral window centered on the sourceframe $\mathrm{N}_{2} \mathrm{D}^{+}(3-2)$ line (rest frequency is $231.32183 \mathrm{GHz} ; \mathrm{C} 1$ source-frame radial velocity is $\left.79.4 \mathrm{~km} \mathrm{~s}^{-1}\right)$, with a total bandwidth of $58 \mathrm{MHz}\left(76 \mathrm{~km} \mathrm{~s}^{-1}\right)$ and a velocity resolution of $0.05 \mathrm{~km} \mathrm{~s}^{-1}$. Baseband 2 was set to a single spectral window for a continuum observation, centered at $231.00 \mathrm{GHz}$, with a total bandwidth of about $2 \mathrm{GHz}$, but also including coverage of ${ }^{12} \mathrm{CO}(2-1)$ (rest frequency $230.538 \mathrm{GHz}$ ) with a velocity resolution of $1.3 \mathrm{~km} \mathrm{~s}^{-1}$. This setup is especially useful for identifying $\mathrm{CO}$ outflows from protostars. (The $\mathrm{CO}$ line is excluded from the continuum band and the final aggregate continuum bandwidth is $\sim 1.4 \mathrm{GHz}$ for imaging.) Baseband 3 was set to a single spectral window centered on $\mathrm{C}^{18} \mathrm{O}(2-1)$ (rest frequency $219.56036 \mathrm{GHz}$ ) with a total bandwidth of $58 \mathrm{MHz}$ $\left(80 \mathrm{~km} \mathrm{~s}^{-1}\right)$ and a velocity resolution of $0.05 \mathrm{~km} \mathrm{~s}^{-1}$. Baseband 4 was split into four spectral windows at rest frequencies of $216.11258 \mathrm{GHz}$ for $\mathrm{DCO}^{+}(3-2), \quad 216.94560 \mathrm{GHz}$ for $\mathrm{CH}_{3} \mathrm{OH}(5(1,4)-4(2,2)), 215.59595 \mathrm{GHz}$ for $\mathrm{SiO}_{v=1}(5-4)$, and $217.23853 \mathrm{GHz}$ for DCN(3-2). Each of these windows has a total bandwidth of $58 \mathrm{MHz}\left(\sim 81 \mathrm{~km} \mathrm{~s}^{-1}\right)$ and a velocity resolution of $0.2 \mathrm{~km} \mathrm{~s}^{-1}$. The data are Hanning smoothed.

We performed the standard cleaning procedure in CASA (Common Astronomy Software Applications) using the task clean. For the continuum and $\mathrm{CO}$ data, we used the Briggs weighting with a robust number of 0.5 (restoring beam $\sim 0$ "! 25 ). To have the best sensitivity, we used the natural weighting for the line data, including $\mathrm{N}_{2} \mathrm{D}^{+}(3-2), \mathrm{DCO}^{+}(3-2), \mathrm{C}^{18} \mathrm{O}(3-2)$, and $\mathrm{DCN}(3-2)$ (restoring beam $\sim 0$ ". 3 ). For the compact data, we performed three iterations of phase-only self-calibration, which improved the sensitivity by a factor of two. The self-calibration solutions to the continuum image were applied to other spectral windows. The final continuum sensitivity achieved is $0.12 \mathrm{mJy} \mathrm{beam}^{-1}$ for the compact-configuration data, $0.042 \mathrm{mJy} \mathrm{beam}^{-1}$ for the extendedconfiguration data, and $0.040 \mathrm{mJy}^{\text {beam }}{ }^{-1}$ for the combined data. The line sensitivity is $\sim 10 \mathrm{mJy}_{\text {beam }}^{-1}$ per $0.1 \mathrm{~km} \mathrm{~s}^{-1}$ for the compact-configuration data, $\sim 8 \mathrm{mJy}^{-1}$ beam $^{-1}$ per $0.1 \mathrm{~km} \mathrm{~s}^{-1}$ for the extended-configuration data, and $\sim 6 \mathrm{mJy}^{\text {beam }}{ }^{-1}$ per $0.1 \mathrm{~km} \mathrm{~s}^{-1}$ for the combined data. In the following, all analyses are based on the combined images.

\subsection{VLA Observations}

The Karl G. Jansky Very Large Array (VLA) of NRAO was pointed toward C1-S in its D configuration on 2014 August 12 and September 11, with the same correlator setup to observe $\mathrm{NH}_{3}(J, K)=(1,1)$ and $(2,2)$ lines. We used a bandwidth of $8 \mathrm{MHz}$ with a channel width of $7.8 \mathrm{kHz}$ (corresponding to $\sim 0.1 \mathrm{~km} \mathrm{~s}^{-1}$ ) in dual polarization. The antenna gain, bandpass, and flux variations were calibrated by standard calibrators $\mathrm{J} 1851+0035$, J1743-0350, and 3C286, respectively, for both observing sessions. Data reduction was performed in CASA 4.2.2. The calibrated visibilities from the two sessions were imaged together. During imaging, we smoothed the channel width to $0.2 \mathrm{~km} \mathrm{~s}^{-1}$ and used a natural weighting in order to optimize the signal-to-noise ratio. The resulting data cubes have an rms noise of $4 \mathrm{mJy}$ per $3^{\prime \prime}$ synthesized beam.

We will use these $\mathrm{NH}_{3}$ data to derive gas kinetic temperature following the routines outlined in Wang et al. (2012, 2014), which use the detailed method developed by Ho \& Townes (1983) and Rosolowsky et al. (2008). We model the $\mathrm{NH}_{3}$ $(J, K)=(1,1)$ and $(2,2)$ spectra simultaneously with five free parameters-kinetic temperature, excitation temperature, $\mathrm{NH}_{3}$ column density, velocity dispersion, and line central velocity. The model fitting is performed on pixels with $>6 \sigma$ integrated intensity for $\mathrm{NH}_{3}(J, K)=(1,1)$. These temperature measurements are discussed below in Section 3.1, specifically for their use in providing partial constraints on the temperatures needed for mass estimates from the millimeter dust continuum emission, assuming coupling of gas and dust temperatures.

\section{Results}

\section{1. $1.3 \mathrm{~mm}$ Dust Continuum Emission and $\mathrm{NH}_{3}$-derived Temperature Map}

Figure 1 shows the $1.30 \mathrm{~mm}$ continuum images of the C1-S region. Note that T16 already presented compact-configuration images of the entire observed region and in this paper we will focus mostly on the subregion shown in Figure 1. Panel (a) shows the compact-array data with 1 !'5 beam. The two protostellar cores $\mathrm{C} 1-\mathrm{Sa}$ and $\mathrm{C} 1-\mathrm{Sb}$ from $\mathrm{T} 16$ are labeled with plus signs. Panel (b) presents the extended-array data with a beam size of 0.2 . Panel (c) shows the combined data with a final resolution also of 0 ". 2 . The synthesized beam corresponds to a physical scale of about $0.005 \mathrm{pc}$, i.e., $\sim 1000 \mathrm{au}$, given the adopted distance to the source of $5.0 \mathrm{kpc}$. Panel (d) shows the same data as (c), but now smoothed to about 0.15 resolution. We also overlay contours for the continuum emission in panel (d). There is a small continuum peak in $\mathrm{C} 1-\mathrm{S}$.

We define $\mathrm{C} 1-\mathrm{Sa}$ and $\mathrm{C} 1-\mathrm{Sb}$ with two circular regions (the dashed circles in Figures 1(c) and (d)). The C1-Sa core shows a relatively smooth profile as a function of radius from the center, resolved with $\gtrsim 10$ beams and with no sign of fragmentation. The bright central region shows elongation in the direction of Galactic longitude. The elongation is resolved with three synthesized beams. In the continuum image of Figure 1(c), a bipolar structure is seen stretching out from $\mathrm{C} 1-\mathrm{Sa}$ in the approximately (Galactic) north-south directions. This structure coincides with the $\mathrm{CO}$ outflow axis (T16, and see Section 3.3 below), indicating that there is dust in this outflow or along its cavity walls that is likely heated to temperatures greater than those of dust in the ambient environment.

Our defined core region of $\mathrm{C} 1-\mathrm{Sb}$ is much smaller than that of C1-Sa, with only $\sim 4$ beams across its diameter. The peak flux density is also several times smaller than that of C1-Sa.

The angular distance between $\mathrm{C} 1-\mathrm{Sa}$ and $\mathrm{C} 1-\mathrm{Sb}$ is $\sim 3^{\prime \prime}$, corresponding to about 15,000 au $(0.073 \mathrm{pc})$. Interestingly, in this region between $\mathrm{C} 1-\mathrm{Sa}$ and $\mathrm{C} 1-\mathrm{Sb}$, we see a diffuse distribution of $1.30 \mathrm{~mm}$ dust emission. At least in projection, it appears to connect $\mathrm{C} 1-\mathrm{Sa}$ and $\mathrm{C} 1-\mathrm{Sb}$ with an arc-like shape. It does not show any clear centrally peaked profile. This structure spatially overlaps with the $\mathrm{N}_{2} \mathrm{D}^{+}(3-2)$ core $\mathrm{C} 1-\mathrm{S}$ defined in T13. In the following, we show the new high-resolution $\mathrm{N}_{2} \mathrm{D}^{+}$data and we make new definitions of the size of this core, 
(a) color:Cycle 2 continuum compact

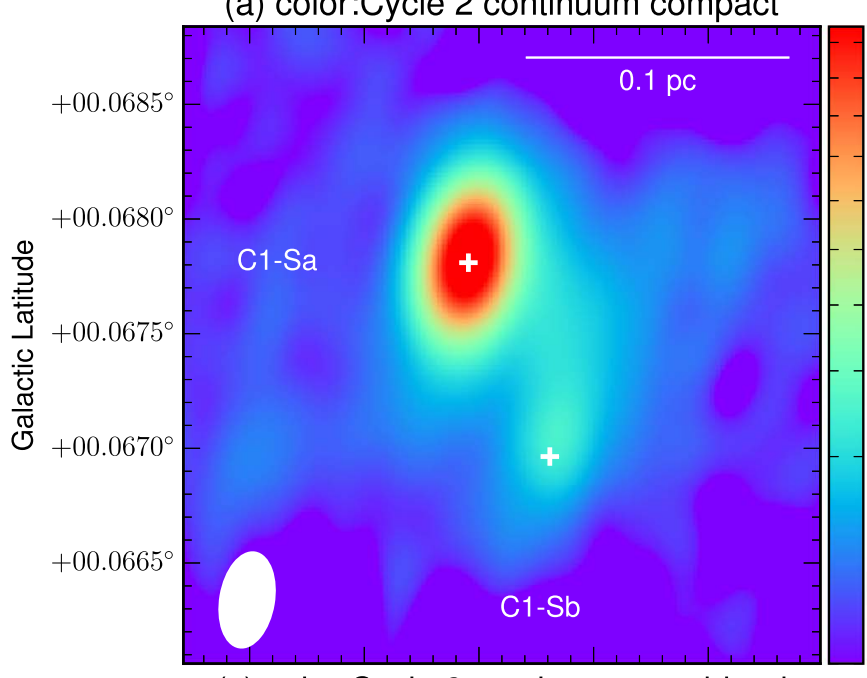

(c) color:Cycle 2 continuum combined

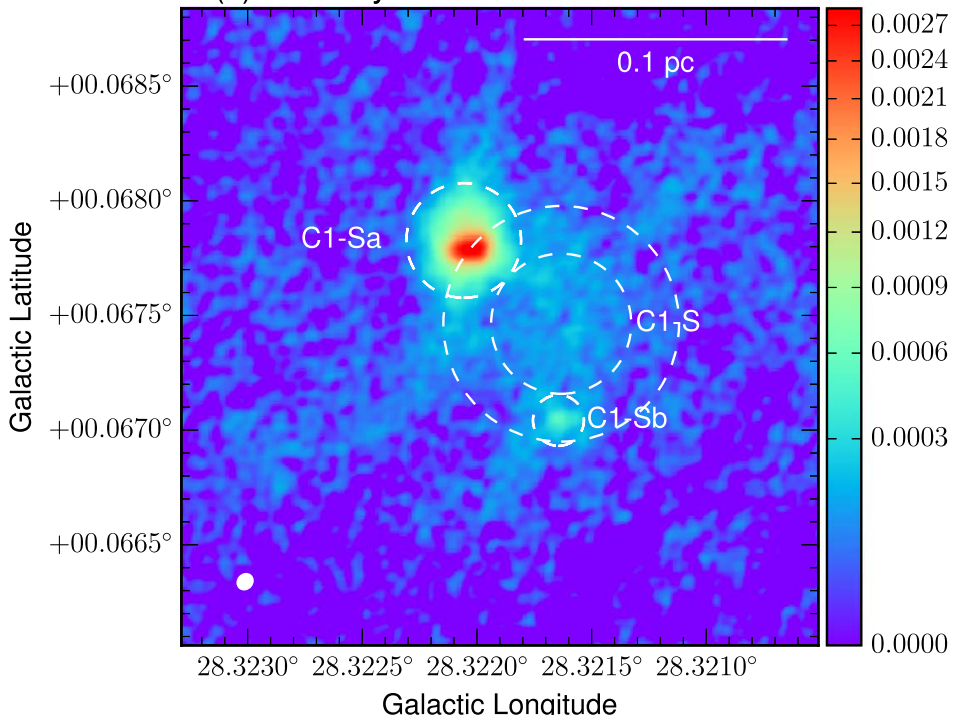

(b) color:Cycle 2 continuum extended

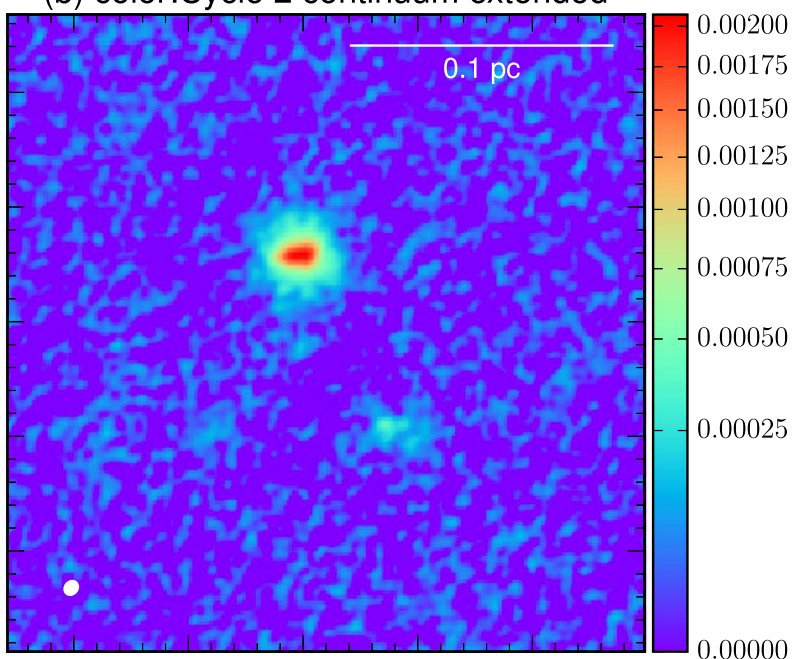

(d) color:Cycle 2 cont. comb. smoothed

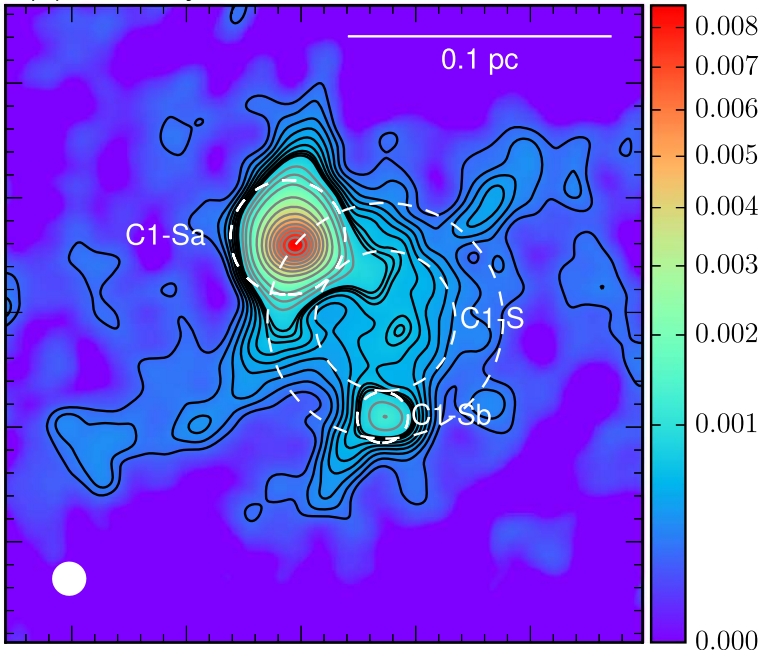

$28.3230^{\circ} 28.3225^{\circ} 28.3220^{\circ} 28.3215^{\circ} 28.3210^{\circ}$

Galactic Longitude

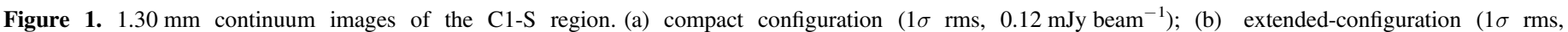

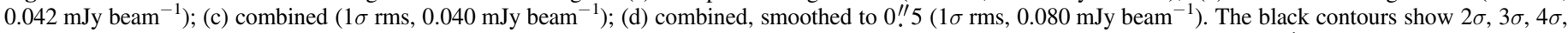

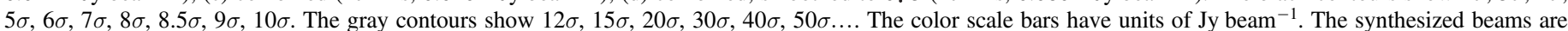

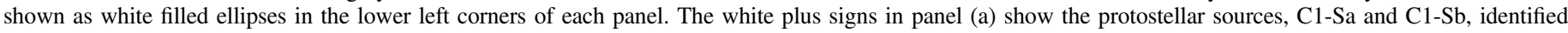

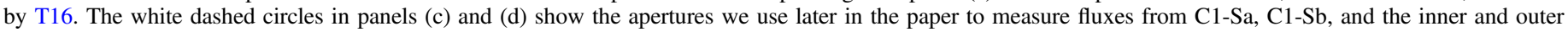
regions of the $\mathrm{C} 1-\mathrm{S}$ candidate massive pre-stellar core.

i.e., with an inner and an outer scale considered, which are also shown in Figures 1(c) and (d).

Our primary method of estimating masses is based on $1.3 \mathrm{~mm}$ dust continuum emission, following Equation (7) of T13, i.e., using the opacities of the model of a moderately coagulated thin ice mantle of Ossenkopf \& Henning (1994). The mass surface density from the millimeter continuum is

$$
\begin{aligned}
\Sigma_{\mathrm{mm}}= & 5.53 \times 10^{-3}\left(\frac{S_{\lambda} / \Omega}{\mathrm{MJy} / \mathrm{sr}}\right)\left(\frac{\kappa_{\lambda}}{0.01 \mathrm{~cm}^{2} \mathrm{~g}^{-1}}\right)^{-1} \lambda_{1.30}^{3} \\
& \times\left[\exp \left(1.106 T_{d, 10}^{-1} \lambda_{1.30}^{-1}\right)-1\right] \mathrm{g} \mathrm{cm}^{-2}
\end{aligned}
$$

where $\lambda_{1.30}=\lambda / 1.30 \mathrm{~mm}$ and $T_{d, 10}=T_{d} / 10 \mathrm{~K}$. We choose $\kappa_{\nu}=5.95 \times 10^{-3} \mathrm{~cm}^{2} \mathrm{~g}^{-1}$ and assume $30 \%$ uncertainties in these opacities. This method of mass estimation depends on the adopted dust temperature, which is somewhat uncertain. We thus calculate core masses for a range of temperatures.
Figure 2 shows the gas kinetic temperature $T_{k}$ measured from the VLA $\mathrm{NH}_{3}$ observations. A temperature of $T_{k} \simeq 13 \mathrm{~K}$ to $16 \mathrm{~K}$ is observed toward the $\mathrm{C} 1-\mathrm{S}$ region, with slightly higher temperatures just to the north. However, given the relatively low critical density $\left(\sim 10^{3} \mathrm{~cm}^{-3}\right)$ of the $\mathrm{NH}_{3}$ transitions (i.e., compared to $\sim 3 \times 10^{6} \mathrm{~cm}^{-3}$ for $\mathrm{N}_{2} \mathrm{D}^{+}(3-2)$ ), we expect that the temperature traced by $\mathrm{NH}_{3}$ is likely influenced by the lower-density envelope material around the $\mathrm{C} 1-\mathrm{S} \mathrm{N}_{2} \mathrm{D}^{+}$core. The $\mathrm{NH}_{3}$ lines are also broader than $\mathrm{N}_{2} \mathrm{D}^{+}(3-2)$, as discussed below. The protostellar cores are not well resolved by the $\mathrm{NH}_{3}$ observations, so the ability to see any warmer gas associated with these protostellar envelopes is compromised by beam dilution.

T13 estimated $T_{d}=10 \pm 3 \mathrm{~K}$, based on the region appearing dark at up to $\sim 100 \mu \mathrm{m}$. Thus for the $\mathrm{C} 1-\mathrm{S}$ starless core, defined in the next subsection, we will adopt $T_{\text {dust }}=10 \pm 3 \mathrm{~K}$. In the models of Zhang et al. (2014) for high-mass protostellar cores and those of Zhang \& Tan (2015) for low-mass cores, the 

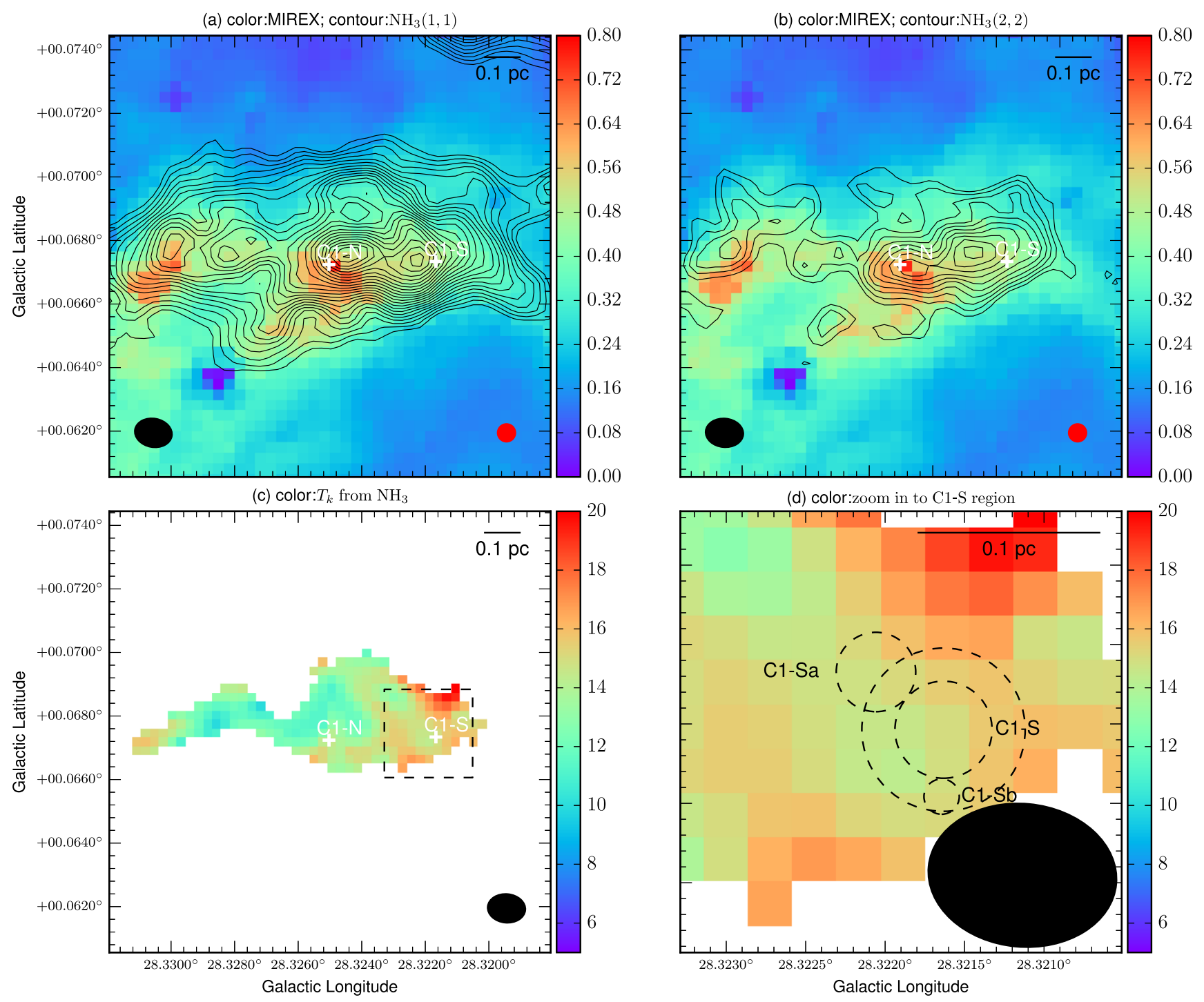

Figure 2. (a) Zeroth-moment map of $\mathrm{NH}_{3}(1,1)$ integrated from 76.9 to $81.9 \mathrm{~km} \mathrm{~s}^{-1}$ (contours, shown from $3 \sigma$, $4 \sigma$, $5 \sigma$, etc., with $\sigma$ being $4 \mathrm{mJy} \mathrm{km} \mathrm{s}{ }^{-1}$ ). The integration covers the main hyperfine structure. The background image shows the mid-IR + near-IR extinction map (in $\mathrm{g} \mathrm{cm}^{-2}$ ) from Butler et al. (2014). The white plus signs represent the C1-N and C1-S cores from T13. The red circle at the lower right shows the resolution beam of the extinction map. The black ellipse at the lower left shows the synthesized beam of the VLA observations. (b) As panel (a), but now showing the zeroth-moment map of $\mathrm{NH}_{3}(2,2)$. (c) $T_{k}$ map derived from the VLA NH 3 observations. The color scale bar has units of kelvin. The filled ellipse is the beam of the VLA observations. The dashed inset shows the region of C1-S enlarged in panel (d). (d) Zoom-in view to the C1-S region in the $T_{k}$ map. The black dashed circles represent the C1-S, C1-Sa, and C1-Sb core definitions (see Sections 3.1 and 3.2.1). The filled ellipse at the lower right is the beam of the VLA observations.

envelope gas temperature is between $\sim 15 \mathrm{~K}$ and $30 \mathrm{~K}$ during the phases of evolution relevant to $\mathrm{C} 1-\mathrm{Sa}$ (i.e., early phases, given the expectation of it being a massive protostellar core, see below) and to $\mathrm{C} 1-\mathrm{Sb}$ (i.e., most evolutionary phases relevant to low-mass star formation, given the expectation that it is a lower-mass protostar, see below). Here we adopt a fiducial temperature of $20 \mathrm{~K}$ for the protostellar cores $\mathrm{C} 1-\mathrm{Sa}$ and $\mathrm{C} 1-\mathrm{Sb}$, with lower and upper bounds of $15 \mathrm{~K}$ and $30 \mathrm{~K}$, respectively. The measured core flux density is $40.9 \mathrm{mJy}$ in $\mathrm{C} 1-\mathrm{Sa}$ and $2.31 \mathrm{mJy}$ in $\mathrm{C} 1-\mathrm{Sb}$, with systematic uncertainties in these absolute values expected to be $\lesssim 10 \%$ so that overall mass uncertainties are dominated by other factors, such as uncertainties in temperature and dust opacity. Table 1 shows the resulting core masses based on the observed millimeter continuum fluxes, given these temperature uncertainties. The $1 \sigma$ continuum mass sensitivity is $0.03 M_{\odot}$ per beam at $T=20 \mathrm{~K}$.
The mass estimates of the C1-S starless core are discussed in the next subsection. The protostellar envelope of $\mathrm{C} 1-\mathrm{Sa}$ has an estimated mass of about $30 M_{\odot}$, with a range of values from $\sim 20$ to $50 M_{\odot}$ given the temperature uncertainties. Based on the momentum flux of its $\mathrm{CO}$ outflow, T16 estimated the mass of the C1-Sa protostar to be $\lesssim 3 M_{\odot}$. Thus C1-Sa is consistent with being a massive protostellar core that is in a relatively early phase of collapse. We find that $\mathrm{C} 1-\mathrm{Sb}$ has about a 14 times smaller millimeter continuum flux and thus the mass estimates for its envelope are just a few solar masses. Given that its $\mathrm{CO}$ outflow momentum flux is only moderately smaller than that of $\mathrm{C} 1-\mathrm{Sa}$, this suggests that $\mathrm{C} 1-\mathrm{Sb}$ is a low- or intermediate-mass protostar at a more advanced evolutionary stage of its collapse. This is also consistent with C1-Sb's larger outflow cavity opening angle (T16, see also Section 3.3). 
Table 1

Core Masses

\begin{tabular}{|c|c|c|c|c|c|c|}
\hline Protostellar Cores & $\begin{array}{c}l \\
(\mathrm{deg})\end{array}$ & $\begin{array}{c}b \\
(\mathrm{deg})\end{array}$ & $\begin{array}{c}R_{\mathrm{c}} \\
(0.01 \mathrm{pc})\end{array}$ & $\begin{array}{c}M_{\mathrm{c}, \mathrm{mm}}(T=15 \mathrm{~K}) \\
\left(M_{\odot}\right)\end{array}$ & $\begin{array}{c}M_{\mathrm{c}, \mathrm{mm}}(T=20 \mathrm{~K}) \\
\left(M_{\odot}\right)\end{array}$ & $\begin{array}{c}M_{\mathrm{c}, \mathrm{mm}}(T=30 \mathrm{~K}) \\
\left(M_{\odot}\right)\end{array}$ \\
\hline C1-Sa & 28.32209 & 0.06770 & 2.18 & 49.5 & $33.5^{\mathrm{a}}$ & 20.2 \\
\hline $\mathrm{C} 1-\mathrm{Sb}$ & 28.32168 & 0.06692 & 0.97 & 3.44 & $2.33^{\mathrm{a}}$ & 1.41 \\
\hline Starless core & $\begin{array}{c}l^{\mathrm{b}} \\
(\mathrm{deg})\end{array}$ & $\begin{array}{c}b^{\mathrm{b}} \\
(\mathrm{deg})\end{array}$ & $\begin{array}{c}R_{\mathrm{c}} \\
(0.01 \mathrm{pc})\end{array}$ & $\begin{array}{c}M_{\mathrm{c}, \mathrm{mm}}(T=7 \mathrm{~K}) \\
\left(M_{\odot}\right)\end{array}$ & $\begin{array}{c}M_{\mathrm{c}, \mathrm{mm}}(T=10 \mathrm{~K}) \\
\left(M_{\odot}\right)\end{array}$ & $\begin{array}{c}M_{\mathrm{c}, \mathrm{mm}}(T=13 \mathrm{~K}) \\
\left(M_{\odot}\right)\end{array}$ \\
\hline $\begin{array}{l}\text { C1-S inner (tot) } \\
\text { C1-S inner }\end{array}$ & $\begin{array}{c}28.32167 \\
\ldots\end{array}$ & $\begin{array}{c}0.06734 \\
\ldots\end{array}$ & $\begin{array}{c}2.67 \\
\ldots\end{array}$ & $\begin{array}{l}53.5 \\
43.7\end{array}$ & $\begin{array}{l}25.5^{\mathrm{a}} \\
17.1^{\mathrm{a}}\end{array}$ & $\begin{array}{l}11.6 \\
4.97\end{array}$ \\
\hline $\begin{array}{l}\text { C1-S outer (tot) } \\
\text { C1-S outer }{ }^{\mathrm{c}}\end{array}$ & $\begin{array}{c}28.32167 \\
\ldots\end{array}$ & $\begin{array}{c}0.06734 \\
\ldots\end{array}$ & $\begin{array}{c}4.48 \\
\ldots\end{array}$ & $\begin{array}{l}123 \\
112\end{array}$ & $\begin{array}{l}58.8^{\mathrm{a}} \\
48.8^{\mathrm{a}}\end{array}$ & $\begin{array}{l}26.8 \\
19.0\end{array}$ \\
\hline
\end{tabular}

Notes.

${ }^{a}$ Fiducial case.

${ }^{b}$ Defined at the center of the circular core.

${ }^{c}$ Masses estimated using total millimeter continuum fluxes.

${ }^{\mathrm{d}}$ Masses estimated using background-subtracted millimeter continuum fluxes.

Table 2

Continuum Flux Measurement Data for Figures 4 and 5

\begin{tabular}{lcccc}
\hline \hline $\begin{array}{l}R_{\mathrm{C} 1-\mathrm{Sa}} \\
(\mathrm{au})\end{array}$ & $\begin{array}{c}\bar{F}_{\mathrm{C} 1-\mathrm{Sa}}(R) \\
\left(\mathrm{MJy} \mathrm{sr}^{-1}\right)\end{array}$ & $\begin{array}{c}R_{\mathrm{C} 1-\mathrm{S} \text { outer }} \\
(\mathrm{au})\end{array}$ & $\begin{array}{c}\bar{F}_{\mathrm{C} 1-\mathrm{S}_{\text {outer }}(R)} \\
\left(\mathrm{MJy} \mathrm{sr}^{-1}\right)\end{array}$ & $\begin{array}{c}\bar{F}_{\mathrm{C} 1-\text { Soutersub }^{-1}}(R) \\
\left(\mathrm{MJy} \mathrm{sr}^{-1}\right)\end{array}$ \\
\hline 588 & $2266(25)$ & 554.5 & $150.9(27.0)$ & $117.7(36.4)$ \\
1205 & $1833(19)$ & 1168.3 & $150.1(19.0)$ & $116.9(30.9)$ \\
1800 & $1249(15)$ & 1757.5 & $151.2(15.8)$ & $118.0(29.0)$ \\
2397 & $798(13)$ & 2349.2 & $136.6(13.4)$ & $103.4(27.7)$ \\
2998 & $522(11)$ & 2949.7 & $126.3(12.0)$ & $93.1(27.1)$ \\
3597 & $346(10)$ & 3549.2 & $130.3(10.9)$ & $97.2(26.6)$ \\
4193 & $231(10)$ & 4146.6 & $126.4(10.1)$ & $93.3(26.3)$ \\
$\ldots$ & $\ldots$ & 4747.3 & $117.6(9.4)$ & $84.4(26.1)$ \\
$\ldots$ & $\ldots$ & 5347.9 & $117.5(8.9)$ & $84.4(25.9)$ \\
$\ldots$ & $\ldots$ & 5940.6 & $106.6(8.9)$ & $73.4(25.9)$ \\
$\ldots$ & $\ldots$ & 6547.4 & $93.4(8.8)$ & $60.3(25.9)$ \\
$\ldots$ & $\ldots$ & 7146.4 & $89.5(8.7)$ & $56.4(25.8)$ \\
$\ldots$ & $\ldots$ & 7743.3 & $79.5(8.3)$ & $46.3(25.7)$ \\
$\ldots$ & $\ldots$ & 8348.5 & $79.8(8.0)$ & $46.6(25.6)$ \\
$\ldots$ & $\ldots$ & 8953.4 & $80.6(7.7)$ & $47.5(25.5)$ \\
\hline
\end{tabular}

(This table is available in machine-readable form.)

\section{2. $\mathrm{N}_{2} D^{+}(3-2)$ Emission}

\subsubsection{Definition of the C1-S Pre-stellar Core}

Figure 3 shows the $\mathrm{N}_{2} \mathrm{D}^{+}(3-2)$ zeroth-moment map of the C1-S region, integrated from 76.9 to $81.9 \mathrm{~km} \mathrm{~s}^{-1}$ (i.e., centered on the $79.4 \mathrm{~km} \mathrm{~s}^{-1}$ measured for the C1-S core by T13). Several versions of the map are shown. In panel (a), we show the combined data of $\mathrm{N}_{2} \mathrm{D}^{+}(3-2)$ with a final synthesized beam size of 0 ". 3 . In panel (b), the same $\mathrm{N}_{2} \mathrm{D}^{+}$image is overlaid as contours on top of the $1.30 \mathrm{~mm}$ continuum image from Figure 1(c). Since noise features are quite visible across the image in panel (a), in panel (c) we show a smoothed image, obtained by applying an outer uv-taper to the $\mathrm{N}_{2} \mathrm{D}^{+}$data to have a $0 . \prime 57 \times 0 . \prime 54$ beam with position angle $89^{\circ} .59$. Then in panel (d), we show the smoothed $\mathrm{N}_{2} \mathrm{D}^{+}$image on top of the continuum image. One can see from Figure 3, especially in panels (c) and (d), that there is centrally concentrated $\mathrm{N}_{2} \mathrm{D}^{+}$ emission in between C1-Sa and C1-Sb. As we discuss below, this structure, which we will refer to as a "core," contains a large fraction of the $\mathrm{N}_{2} \mathrm{D}^{+}(3-2)$ flux of the C1-S core defined by T13. We recall that the $\mathrm{N}_{2} \mathrm{D}^{+}(3-2)$ flux of $\mathrm{C} 1-\mathrm{S}$ is the strongest source of such emission in the wider region and is highly localized in position and velocity space, i.e., to $\sim 0.1 \mathrm{pc}$ spatial and $\lesssim 0.5 \mathrm{~km} \mathrm{~s}^{-1}$ velocity scales. In addition to the radial gradient in $\mathrm{N}_{2} \mathrm{D}^{+}(3-2)$ intensity, $\mathrm{C} 1-\mathrm{S}$ also shows some internal substructure in this emission at the level of a factor of about two to three. Such variation is broadly consistent with the astrochemical models of Goodson et al. (2016) of a monolithic core that is partially supported by turbulence. In these models the abundance of $\mathrm{N}_{2} \mathrm{D}^{+}$increases by several orders of magnitude and depends on local density, temperature, and other gas properties, so fluctuations of a factor of a few are readily explained as being in response to local transient turbulent fluctuations, without necessarily implying that these structures will fragment into separate entities.

We define "C1-S inner" as the circle centered approximately on the peak $\mathrm{N}_{2} \mathrm{D}^{+}(3-2)$ zeroth-moment contours, while extending out as far as possible to just reach, in projection, the C1-Sa and C1-Sb protostellar cores. C1-S inner contains $23 \%$ of the total $\mathrm{N}_{2} \mathrm{D}^{+}(3-2)$ flux in the high-resolution image (panel (b)). We also define "C1-S outer" with a circular aperture that shares the same center but is expanded to cover a larger fraction (44\%) of the $\mathrm{N}_{2} \mathrm{D}^{+}(3-2)$ flux in the region (see Figures 3(b) and (d)). C1-S outer partially overlaps C1-Sa and fully overlaps $\mathrm{C} 1-\mathrm{Sb}$. In any later analysis, pixels in C1-Sa and $\mathrm{C} 1-\mathrm{Sb}$, e.g., containing millimeter continuum flux or $\mathrm{N}_{2} \mathrm{D}^{+}(3-2)$ flux, are excluded from contributing to $\mathrm{C} 1-\mathrm{S}$ outer.

Note here that we are defining C1-S inner and outer by their centrally peaked $\mathrm{N}_{2} \mathrm{D}^{+}$emission, unlike $\mathrm{C} 1-\mathrm{Sa}$ or $\mathrm{C} 1-\mathrm{Sb}$ that were defined by centrally peaked continuum emission. As shown in Figure 4 (plot data available in Table 2), C1-Sa shows a centrally peaked $1.3 \mathrm{~mm}$ continuum intensity profile, while C1S's profile is much shallower. The lack of a centrally peaked continuum morphology in C1-S, compared to C1-Sa and C1-Sb, is thus evidence for it being a starless pre-stellar core rather than a protostellar core. Note also that the extent of material that is dynamically associated with C1-S, i.e., bound to it, may be larger than the example aperture of C1-S outer. Figure 3(c) shows this more extended $\mathrm{N}_{2} \mathrm{D}^{+}(3-2)$ emission, although a small part of this may be associated with the $\mathrm{C} 1-\mathrm{Sa}$ and $\mathrm{C} 1-\mathrm{Sb}$ protostars.

The $1.3 \mathrm{~mm}$ continuum-derived mass estimates for $\mathrm{C} 1-\mathrm{S}$ inner and outer shown in Table 1 range from about 25 to $60 M_{\odot}$ 
(a) color: $\mathrm{N}_{2} \mathrm{D}^{+}$

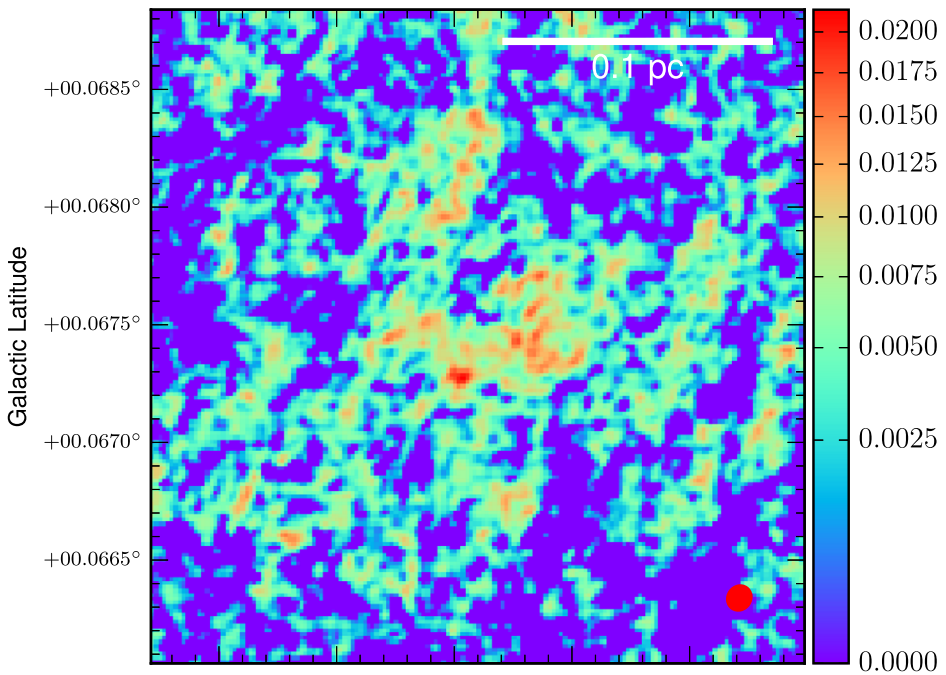

(c) color: $\mathrm{N}_{2} \mathrm{D}^{+}$uvtaper

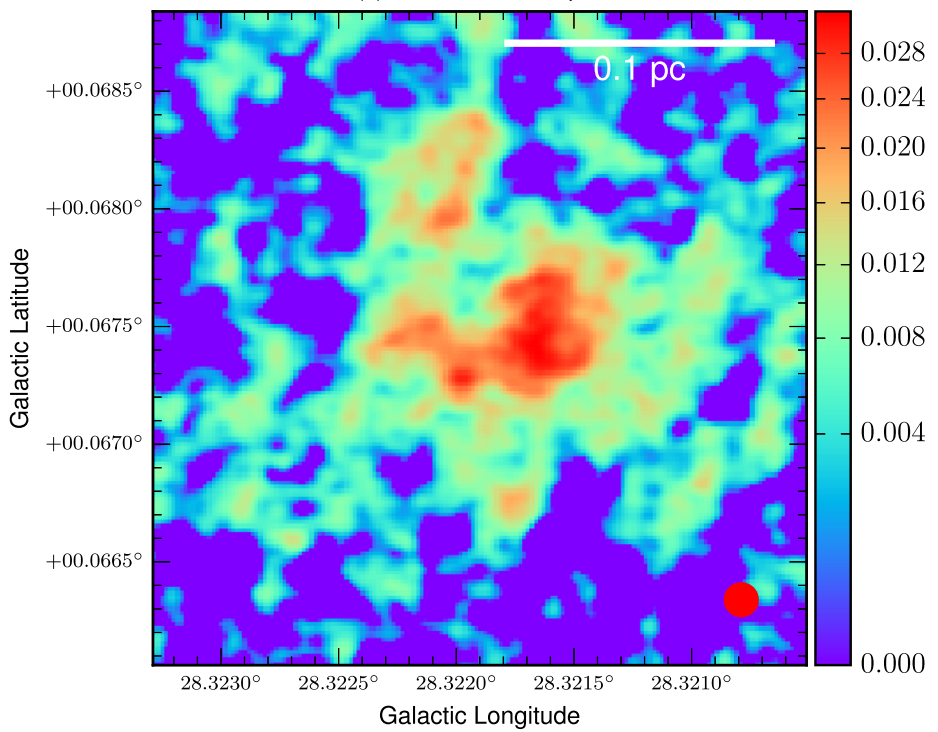

(b) color:1.3 mm continuum; contour: $\mathrm{N}_{2} \mathrm{D}^{+}$

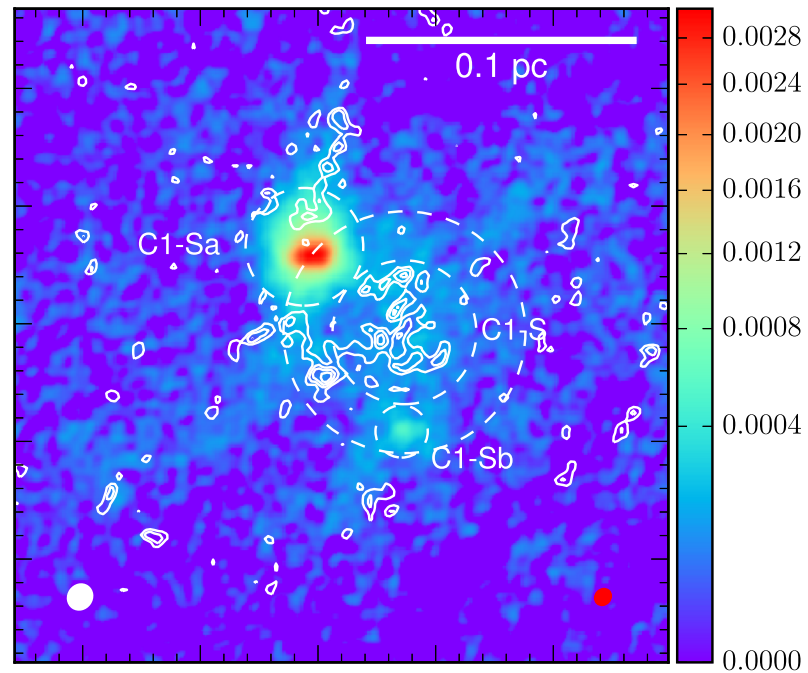

(d) color:1.3 mm continuum; contour: $\mathrm{N}_{2} \mathrm{D}^{+}$uvtaper

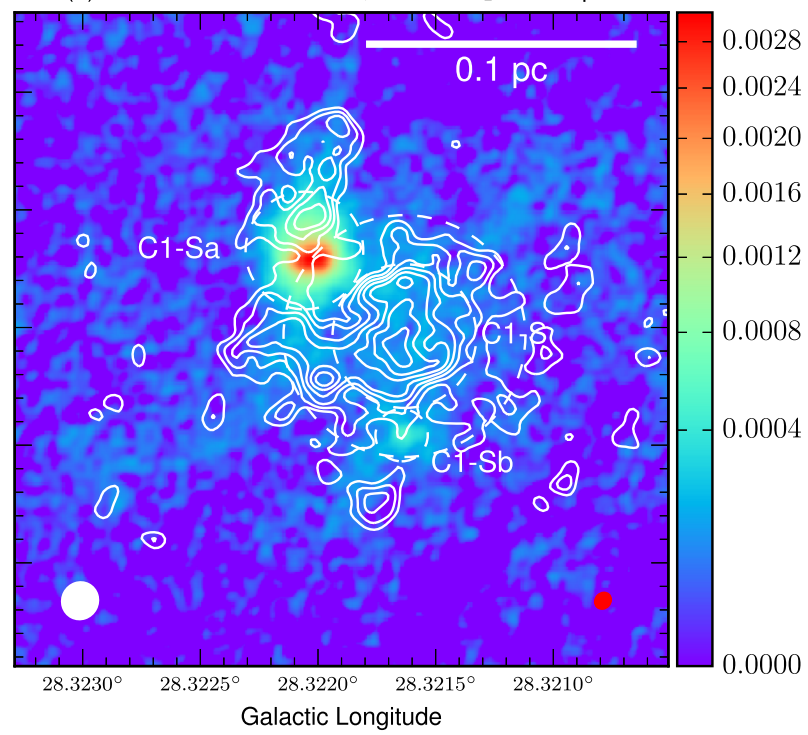

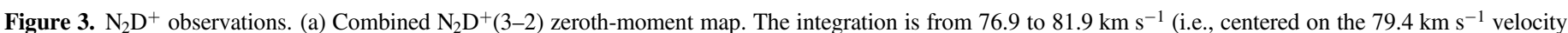

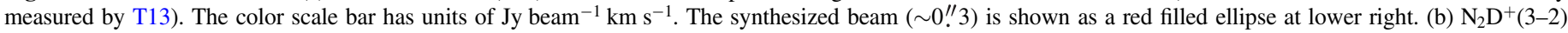

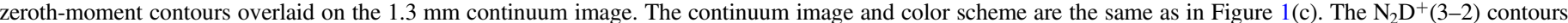

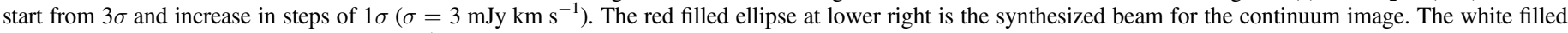

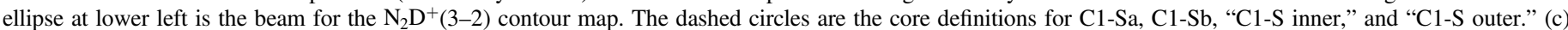

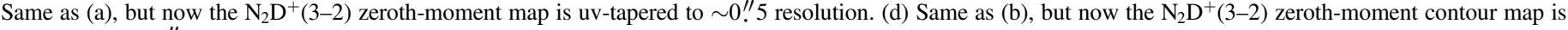
uv-tapered to $\sim 0$." 5 resolution.

for the fiducial choice of assumptions, i.e., a dust temperature of $10 \mathrm{~K}$. Temperature uncertainties from 7 to $13 \mathrm{~K}$ induce about a factor-of-two uncertainty in the mass estimates. Note, however, that these mass estimates have so far been based on the total flux observed toward a given aperture. Clump envelope subtraction, i.e., where the average flux density in an annular region extending to twice the core radius (and excluding the protostars $\mathrm{C} 1-\mathrm{Sa}$ and $\mathrm{C} 1-\mathrm{Sb}$ ) is subtracted (see Section 3.2.4), leads to modest reductions in these mass estimates by factors of about 0.64 for $\mathrm{C} 1-\mathrm{S}$ inner and 0.83 for $\mathrm{C} 1-\mathrm{S}$ outer in the fiducial case. Thus, the mass that is associated with the observed $\mathrm{N}_{2} \mathrm{D}^{+}(3-2)$ emission, especially when considered at its outer scale, appears to be of sufficient quantity to form a massive star, if this core were to undergo quasimonolithic collapse.

C1-S is a highly deuterated core. The deuteration analysis of Kong et al. (2016), which was applied to the C1-S aperture of
3!" 5 radius of T13, found $D_{\text {frac }}^{\mathrm{N} 2 \mathrm{H}+} \equiv\left[\mathrm{N}_{2} \mathrm{D}^{+}\right] /\left[\mathrm{N}_{2} \mathrm{H}^{+}\right] \simeq 0.2$ to 0.7. We thus expect the actual deuteration level in $\mathrm{C} 1-\mathrm{S}$ outer and inner to be even higher. Such high levels of deuteration are expected to be achieved in gas that is kept in a cold $(T \lesssim 20 \mathrm{~K})$ and dense state for a relatively long time, i.e., compared to the local free-fall time. Such conditions are found to be common in low-mass pre-stellar cores (Caselli \& Ceccarelli 2012) and may be expected to arise in more massive pre-stellar cores also, assuming such cores exist.

In summary, $\mathrm{C} 1-\mathrm{S}$ shows no obvious sign of star formation. It has no centrally peaked continuum profile. It has no corresponding $\mathrm{CO}$ outflows (as will be shown below in Section 3.3). In Herschel $70 \mu \mathrm{m}$ and $100 \mu \mathrm{m}$ images, this entire region has no sign of point sources (Lim et al. 2016; W. Lim et al. 2018, in preparation). While $\mathrm{C} 1-\mathrm{Sa}$ and $\mathrm{C} 1-\mathrm{Sb}$ have some level of $\mathrm{N}_{2} \mathrm{D}^{+}(3-2)$ emission, this tracer does not show obvious concentrations at the locations of their continuum emission 


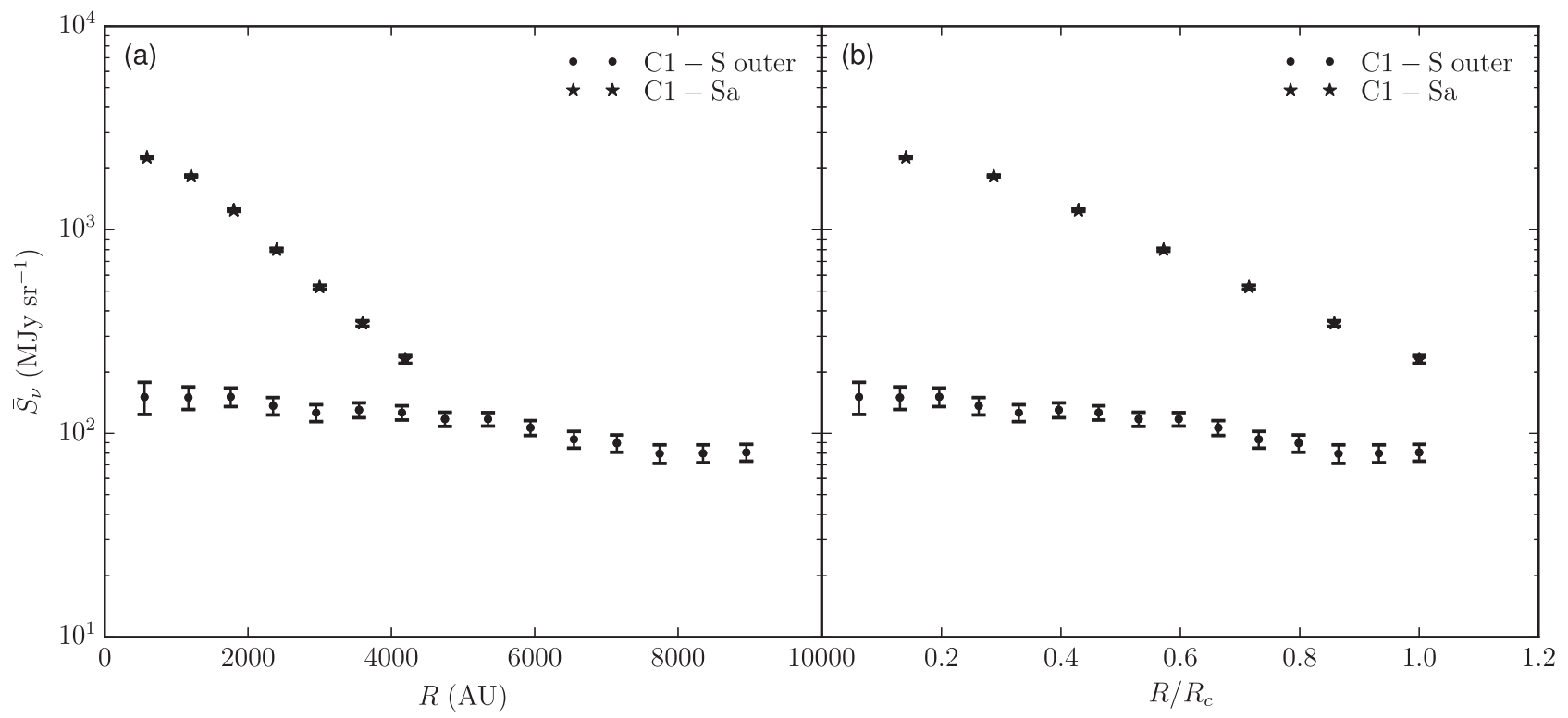

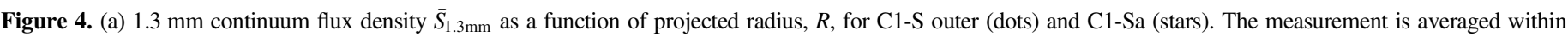

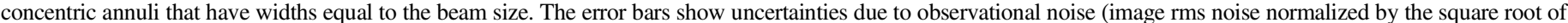

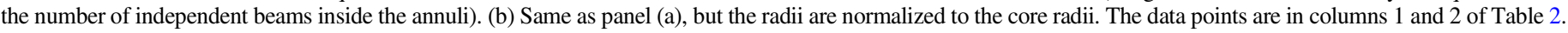

peaks. Meanwhile, C1-S has a strongly concentrated $\mathrm{N}_{2} \mathrm{D}^{+}(3-2)$ structure away from the protostars. We thus conclude that $\mathrm{C} 1-\mathrm{S}$ is likely to be a highly deuterated massive starless core.

\subsubsection{Core Structure}

Figure 5 shows the radial structure of $\mathrm{C} 1-\mathrm{S}$ outer as inferred from thermal dust continuum emission (plot data available in Table 2). In panel (a) we show the total $\bar{S}_{1.3 \mathrm{~mm}}(R)$ (averaged in annuli), while in (b) we show the result after envelope subtraction. This background envelope level is assessed as the average value of $\bar{S}_{1.3 \mathrm{~mm}}$ in an annulus from one to two core radii (excluding the $\mathrm{C} 1-\mathrm{Sa}$ and $\mathrm{C} 1-\mathrm{Sb}$ regions). We estimate the uncertainty associated with this process as equal to the level of fluctuations seen in eight independent regions of the annulus, i.e., regions that have linear dimensions comparable to the radius of the core. The standard deviation of these mean values is adopted as the uncertainty, which is about $70 \%$ of the overall envelope value. In panel (c), we calculate the mass surface density profile from the total $1.3 \mathrm{~mm}$ flux density based on Equation (1). Since C1-S is a candidate starless core, we first assume $T_{\text {dust }}=10 \mathrm{~K}$ across the core (black dots). We also calculate two more cases where $T_{\text {dust }}=7 \mathrm{~K}$ and $T_{\text {dust }}=13 \mathrm{~K}$, which we use to assess the uncertainty in the estimates of mass surface density.

Following the turbulent core model of MT03, we assume a core volume density profile following a singular polytropic spherical structure: $\rho \propto r^{-k_{\rho}}$, where $k_{\rho}=1.5$ in the fiducial case. We project the volume density to surface density $\Sigma$ by integrating along the line of sight. Then we find the best fitting model, first for the case of $k_{\rho}=1.5$ (black line in panel (c)). It has a density at core surface $(9250 \mathrm{au}$, i.e., the radius of $\mathrm{C} 1-\mathrm{S}$ outer) of $n_{H}=8.40 \times 10^{5} \mathrm{~cm}^{-3}$. However, this model is not a very good fit to the data. We then allow $k_{\rho}$ to vary, which yields a best-fit model with $k_{\rho}=-0.215$ (blue line), i.e., $\rho$ increasing toward the core surface, which is not expected for physically realistic models. We repeat the analysis for the profiles with the clump envelope subtracted shown in panel (d). Now the best-fit $k_{\rho}$ is 0.283 . This has density declining with radius, but is still much shallower than the fiducial case of MT03.

In panels (e) and (f), we also investigate models with a temperature gradient in the core. As an example, we consider a linear gradient with $T=7 \mathrm{~K}$ at the core center and $T=13 \mathrm{~K}$ at the core surface, similar to the range of values seen in some lower-mass pre-stellar cores (Crapsi et al. 2007). The newly derived $\Sigma$ profile are shown as dots. One can see that $\Sigma$ is more centrally peaked than in the isothermal case. Again we model the core and the best-fit $k_{\rho}=0.414$ for the total-flux case and $k_{\rho}=0.717$ for the envelope-subtracted case. Opacity variations within the core, e.g., due to grain growth in the denser central regions, could also lead to changes in the derived density structures. Lacking any good constraints on variations in temperature and opacity within the core, we conclude that it is difficult to determine the core structure from the current data. We also note that as a starless core contracts to become a prestellar core on the verge of forming a protostar, its density profile is expected to steepen (Shu 1977).

\subsubsection{Core Kinematics}

Figure 6 shows the spectra of $\mathrm{N}_{2} \mathrm{D}^{+}(3-2), \mathrm{DCO}^{+}(3-2)$, $\mathrm{C}^{18} \mathrm{O}(2-1)$, and $\mathrm{DCN}(3-2)$ from the $\mathrm{C} 1-\mathrm{Sa}, \mathrm{C} 1-\mathrm{Sb}$, and $\mathrm{C} 1-\mathrm{S}$ inner cores. The spectra are averaged over the circular core apertures and converted to main-beam temperature $T_{\mathrm{mb}}$. Considering the spectra of the $\mathrm{C} 1-\mathrm{Sa}$ protostar, one can see that most of its emission from dense gas tracers is close to the $79.4 \mathrm{~km} \mathrm{~s}^{-1}$ velocity component identified in $\mathrm{N}_{2} \mathrm{D}^{+}(3-2)$ by $\mathrm{T} 13$ (hereafter $v_{\text {low }}$ ). There is a relatively weak velocity component in $\mathrm{C}^{18} \mathrm{O}(2-1)$ at $81.2 \mathrm{~km} \mathrm{~s}^{-1}$ (hereafter $v_{\text {high }}$ ). Like T16, we argue that the protostellar core $\mathrm{C} 1-\mathrm{Sa}$ is at the velocity $v_{\text {low }}$. We note that multiple velocity components are commonly seen in IRDCs (e.g., Henshaw et al. 2013).

For the $\mathrm{C} 1-\mathrm{Sb}$ protostar, we see from the spectra of $\mathrm{DCO}^{+}(3-2)$ and $\mathrm{C}^{18} \mathrm{O}(2-1)$ that the main velocity component is at $81.2 \mathrm{~km} \mathrm{~s}^{-1}\left(v_{\text {high }}\right)$. There is a relatively weak component in $\mathrm{DCO}^{+}(2-1)$ at $v_{\text {low }}$ and a hint of a feature in $\mathrm{N}_{2} \mathrm{D}^{+}(3-2)$ at $v_{\text {low }}$. We argue that the protostellar core $\mathrm{C} 1-\mathrm{Sb}$ is at $v_{\text {high }}$. T16 also 


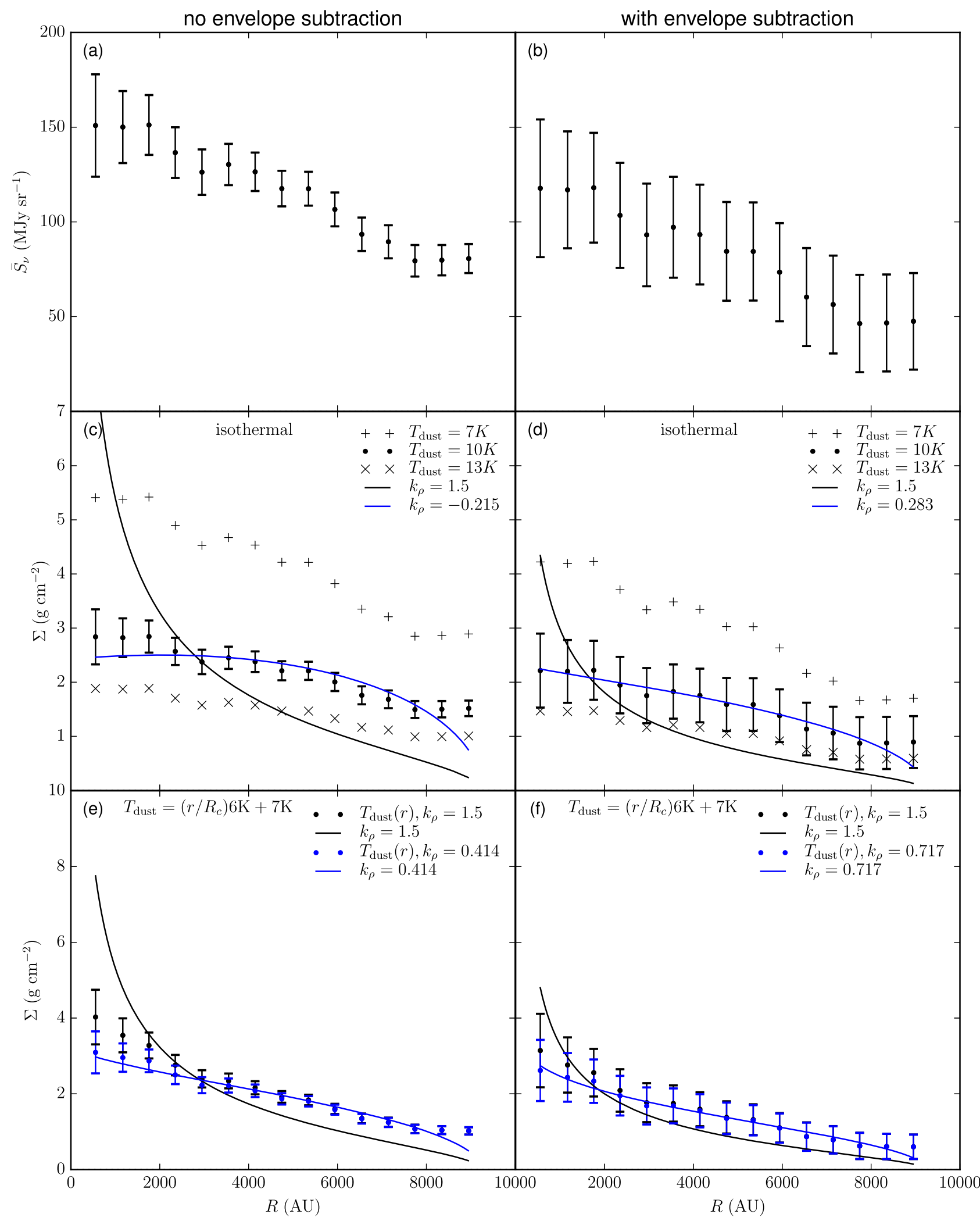

Figure 5. (a) $1.3 \mathrm{~mm}$ continuum flux density $\bar{S}_{1.3 \mathrm{~mm}}$ as a function of projected radius, $R$, for C1-S outer. The measurement is averaged within concentric annuli that have widths equal to the beam size. The error bars show uncertainties due to observational noise. (b) Same as panel (a), but after subtraction of the clump envelope, as evaluated from an annulus from $R_{c}$ to $2 R_{c}$. (c) Mass surface density, $\Sigma$, as a function of projected radius $R$ for C1-S outer assuming isothermal conditions. The fiducial dust temperature is $10 \mathrm{~K}$ (black dots). Also shown are cases with $7 \mathrm{~K}$ and $13 \mathrm{~K}$. The solid lines show $\Sigma$ profiles of model cores with power-law volume density profiles $\rho \propto r^{-k_{\rho}}$, with the best-fit case of $k_{\rho}=-0.215$ shown with a blue line and the fiducial reference case of $k_{\rho}=1.5$ shown with a black line. The normalization of these profiles is based on a $\chi^{2}$ minimization to the data. (d) Same as panel (c), but with clump envelope subtracted. The best-fit $k_{\rho}=0.283$. (e) A linear temperature profile with 3D radius from $7 \mathrm{~K}$ (at core center) to $13 \mathrm{~K}$ (at core surface) is adopted for estimation of the mass surface density. Two $\Sigma$ profiles are shown for the fiducial MT03 turbulent core $\left(k_{\rho}=1.5\right)$ and for the best-fit value of $k_{\rho}$. The black curve is the model for $k_{\rho}=1.5$, and the blue curve is the model involving $k_{\rho}$, which gives the best-fit $k_{\rho}=0.414$. (f) Same as panel (e), but with clump envelope subtracted. The best-fit $k_{\rho}=0.717$. The data points are in columns 3,4 , and 5 of Table 2 . 

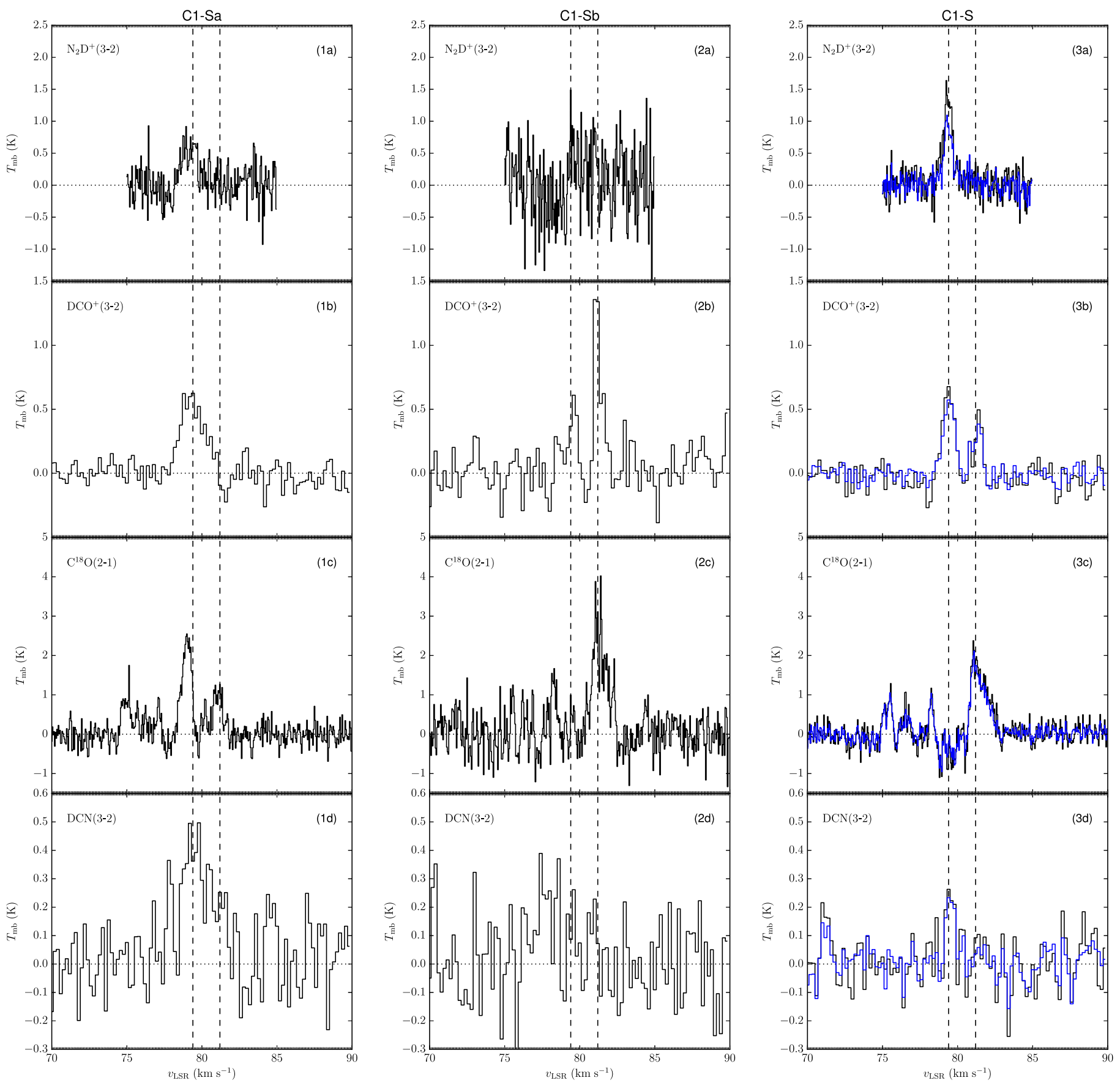

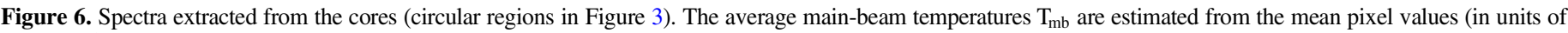

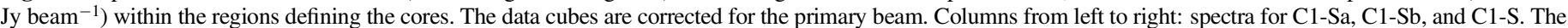

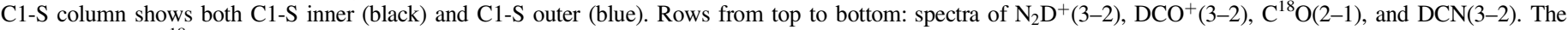

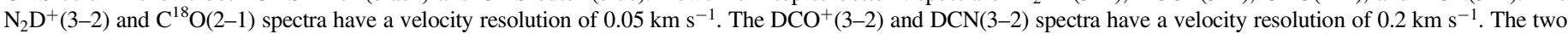

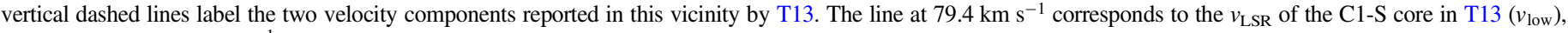
while the line at $81.2 \mathrm{~km} \mathrm{~s}^{-1}$ corresponds to the $\mathrm{C} 1-\mathrm{N}$ core in $\mathrm{T} 13\left(v_{\text {high }}\right)$. The spectra of $\mathrm{C} 1-\mathrm{Sb}$ are relatively noisy because of its small size.

suggested a higher radial velocity for $\mathrm{C} 1-\mathrm{Sb}$ based on the channel maps of ${ }^{12} \mathrm{CO}(2-1)$ with low velocity resolution.

The $\mathrm{C} 1-\mathrm{S}$ core, defined by $\mathrm{N}_{2} \mathrm{D}^{+}(3-2)$, is at $79.4 \mathrm{~km} \mathrm{~s}^{-1}$ $\left(v_{\text {low }}\right)$. This $\mathrm{C} 1-\mathrm{S}$ component is also seen in $\mathrm{DCO}^{+}(3-2)$ and $\operatorname{DCN}(3-2)$, but not in $\mathrm{C}^{18} \mathrm{O}(2-1)$. We also see the $v_{\text {high }}$ component in $\mathrm{DCO}^{+}(3-2)$ and $\mathrm{C}^{18} \mathrm{O}(2-1)$.

Overall, these spectra suggest that $\mathrm{C} 1-\mathrm{Sa}$ is likely to be part of the same cloud enclosing $\mathrm{C} 1-\mathrm{S}$, while $\mathrm{C} 1-\mathrm{Sb}$ is a core that is part of a separate larger-scale structure, potentially connected to C1-N (T13). We note that in the C1 region, we generally see these two velocity components in a variety of data sets. The velocity difference is typically $1.8 \mathrm{~km} \mathrm{~s}^{-1}$. It remains to be seen whether these components are interacting, i.e., via a collision, or are simply overlapping in the plane of the sky but physically separated in the third spatial dimension.

Figures 7 and 8 show the channel maps of the $\mathrm{N}_{2} \mathrm{D}^{+}(3-2)$ data, with the latter uv-tapered to $\sim 0$ ". 5 resolution. The three cores $\mathrm{C} 1-\mathrm{Sa}, \mathrm{C} 1-\mathrm{Sb}$, and $\mathrm{C} 1-\mathrm{S}$ are shown with white dashed circles. The velocity range of the channels covers essentially all of the detected $\mathrm{N}_{2} \mathrm{D}^{+}(3-2)$ emission (above $3 \sigma$ ). $\mathrm{N}_{2} \mathrm{D}^{+}(3-2)$ in 

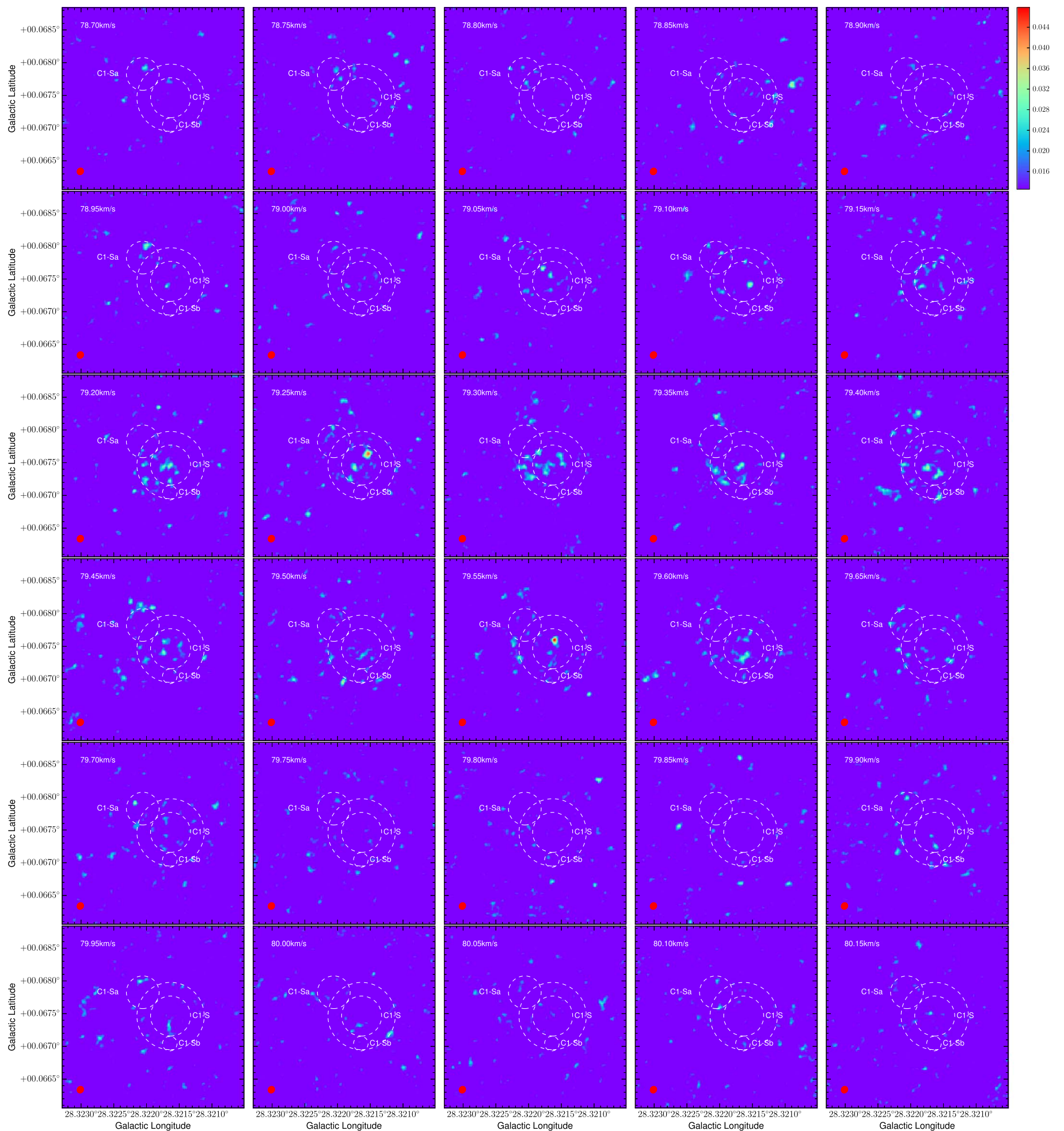

Figure 7. Channel maps of the $\mathrm{N}_{2} \mathrm{D}^{+}(3-2)$ highest-resolution data of the $\mathrm{C} 1$ region (the same as shown in Figure 1; core apertures are marked with dashed circles). The channel velocity is shown in the upper left corner of each panel. The color scheme is from $2 \sigma$ (in this case $\sigma=0.011 \mathrm{Jy}^{-1}$ beam ${ }^{-1}$ ) to the maximum with a linear scale. The color bar has units of Jy beam ${ }^{-1}$. The synthesized beam is shown as a red filled ellipse at lower left.

C1-Sa is more prominent in channels $\lesssim 79.1 \mathrm{~km} \mathrm{~s}^{-1}$, while in C1-S it is more dominant in channels $\gtrsim 79.1 \mathrm{~km} \mathrm{~s}^{-1}$. From $79.1 \mathrm{~km} \mathrm{~s}^{-1}$ to $80.0 \mathrm{~km} \mathrm{~s}^{-1}$, C1-S is filled with widespread $\mathrm{N}_{2} \mathrm{D}^{+}$features, with little indication of any large-scale velocity gradients.

To examine this further, Figure 9 shows the first-moment map of the combined $\mathrm{N}_{2} \mathrm{D}^{+}(3-2)$ data, with velocity measurements shown for those pixels with a $2 \sigma$ and $3 \sigma$ detection of the species, i.e., to illustrate the effect of the choice of this threshold. C1-S shows a quite coherent structure around $79.4 \mathrm{~km} \mathrm{~s}^{-1}$. No clear sign of global rotation is seen in C1-S. There is a stronger velocity gradient seen across $\mathrm{C} 1$-Sa, equivalent to about a $0.8 \mathrm{~km} \mathrm{~s}^{-1}$ change across the diameter of the core (see Figures 9(j) and (n)). The 

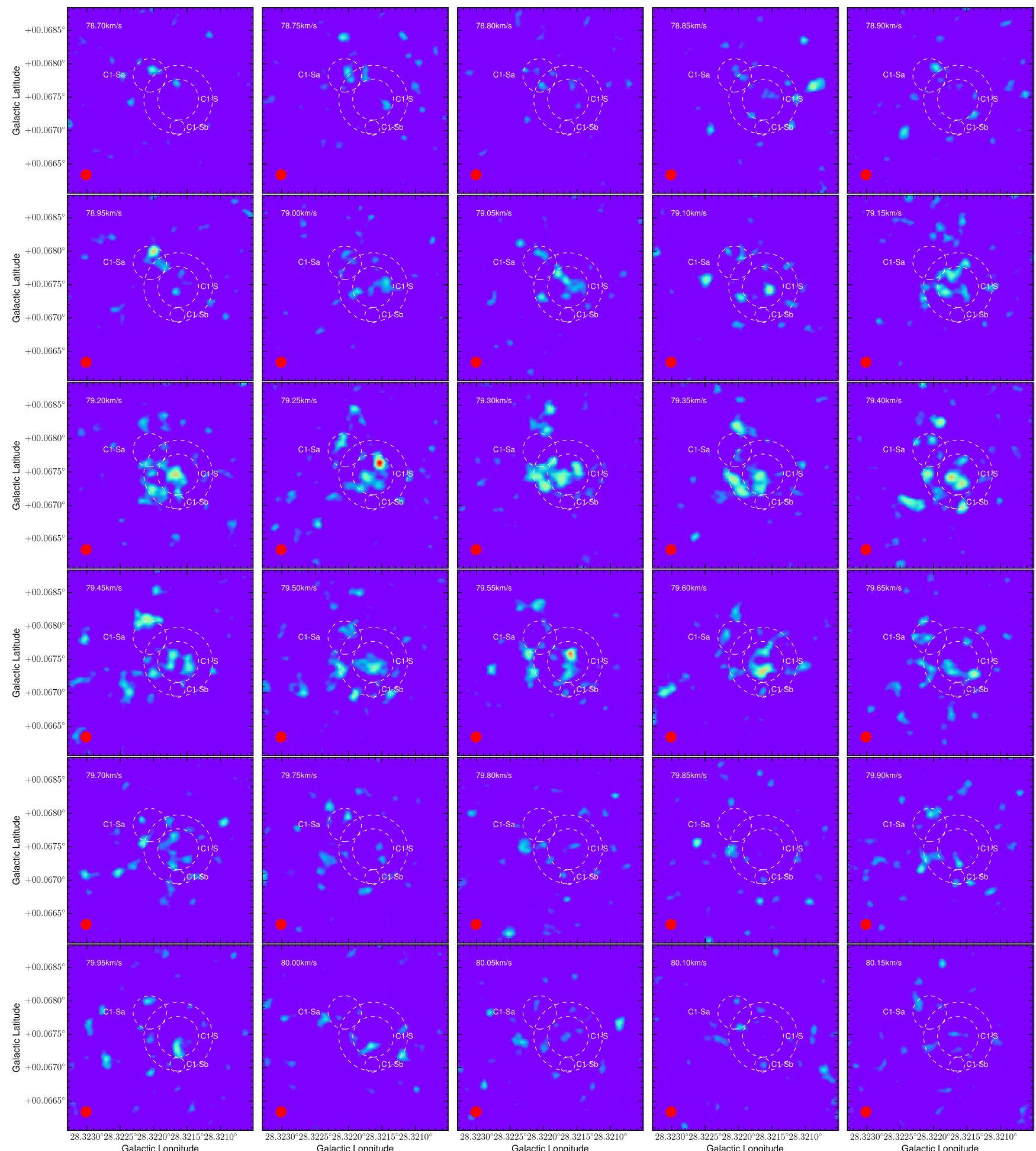

Figure 8. Same as Figure 7, but with the $\mathrm{N}_{2} \mathrm{D}^{+}(3-2)$ data uv-tapered to $\sim 0$." 5 resolution. In this case, $\sigma=0.008 \mathrm{Jy}$ beam $^{-1}$.

direction of the gradient $(\mathrm{E}-\mathrm{W})$ is consistent with the continuum elongation. Figure 9 shows the second-moment map of the combined $\mathrm{N}_{2} \mathrm{D}^{+}$data (the last two columns). In the fourth column, we subtract the intrinsic dispersion of the $\mathrm{N}_{2} \mathrm{D}^{+}(3-2)$ hyperfine structure in quadrature from the secondmoment map (calculated at $2 \sigma$ and $3 \sigma$ ). Consequently, the number of available pixels is limited by the sensitivity. C1-Sa shows a moderately enhanced second moment, which may be caused by its outflow (Section 3.3).
We performed hyperfine-structure spectral fitting to the $\mathrm{N}_{2} \mathrm{D}^{+}(3-2)$ spectrum using the hyperfine structure (HFS) method of the CLASS software package ${ }^{8}$ in Figure 10, assuming that the line is optically thin (see the optical depth check in Kong et al. 2016). The thermal component of $\mathrm{N}_{2} \mathrm{D}^{+}$is subtracted in quadrature from the line dispersion, and the thermal component of $\mathrm{H}_{2}$ gas (assuming $10 \pm 3 \mathrm{~K}$ ) is added

\footnotetext{
8 http://www.iram.fr/IRAMFR/GILDAS
} 

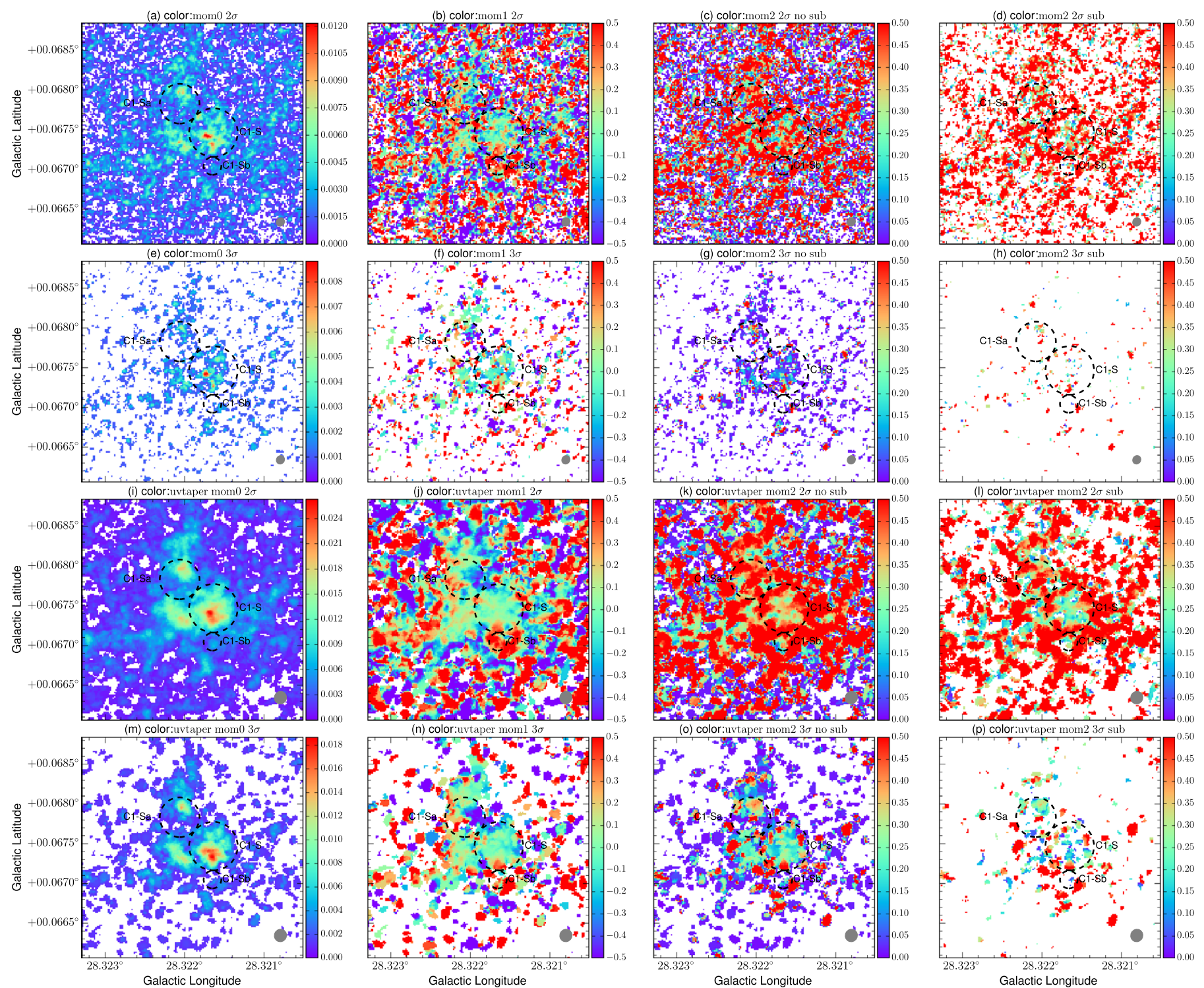

Figure 9. $\mathrm{N}_{2} \mathrm{D}^{+}(3-2)$ moment maps. Columns from left to right: zeroth moment, first moment (relative to $79.4 \mathrm{~km} \mathrm{~s}^{-1}$ ), second moment without subtraction of intrinsic dispersion, and second moment with the dispersion of the $\mathrm{N}_{2} \mathrm{D}^{+}(3-2)$ hyperfine structure subtracted. Rows from top to bottom: combined data with $2 \sigma$ cut, combined data with $3 \sigma$ cut, uv-tapered data $\left(0^{\prime \prime} 5\right)$ with $2 \sigma$ cut, and uv-tapered data with $3 \sigma$ cut. All moment calculations are carried out within the velocity range $79.4 \pm 1.5 \mathrm{~km} \mathrm{~s}^{-1}$ to avoid any contribution from the $81.2 \mathrm{~km} \mathrm{~s}^{-1}$ component. The subtraction of the intrinsic dispersion of $0.242 \mathrm{~km} \mathrm{~s}^{-1}$ from the $\mathrm{N}_{2} \mathrm{D}^{+}(3-2)$ hyperfine structure in the fourth column limits the number of available pixels in the final image. The beam is shown as the gray filled ellipse at the lower right corner. The color bars for the first column have units of Jy beam ${ }^{-1} \mathrm{~km} \mathrm{~s}^{-1}$, and for the other columns it is $\mathrm{km} \mathrm{s}^{-1}$.

back in quadrature to obtain the total velocity dispersion of the core as derived from the $\mathrm{N}_{2} \mathrm{D}^{+}$observations $\left(\sigma_{\mathrm{N}_{2} \mathrm{D}^{+}}\right)$(see T13). These results are listed in Table 3. The observed line profiles of $\mathrm{N}_{2} \mathrm{D}^{+}$are dominated by turbulent motions, although the total velocity dispersions are under $0.4 \mathrm{~km} \mathrm{~s}^{-1}$.

Figure 11 shows the $\mathrm{C} 1-\mathrm{S}$ outer core velocity dispersion $\sigma$ as a function of projected radius, $R$. The core is dissected into the same set of annuli as in Figure 5. Within each annulus, we extract the averaged $\mathrm{N}_{2} \mathrm{D}^{+}(3-2)$ flux density and form a spectrum. Then we fit the hyperfine structure of $\mathrm{N}_{2} \mathrm{D}^{+}(3-2)$ and derive $\sigma_{\mathrm{N}_{2} \mathrm{D}^{+}}$, as described above.

We first model the core velocity dispersion following the MT03 model, where the core volume density follows $\rho(r) \propto r^{-k_{\rho}}$ and the velocity dispersion follows $\sigma(r) \propto r^{k_{\sigma}}$. The fiducial MT03 model has $k_{\rho}=1.5$ and $k_{\sigma}=0.25$. We project $\sigma(r)$ to the sky plane by summing the line-of-sight mass-weighted $\sigma(r)$ in quadrature (this is the same as the emission-weighted averaging in the optically thin, isothermal limit). Finally we normalize the $2 \mathrm{D} \sigma-R$ model based on a $\chi^{2}$ minimization to the data (converted to $\sigma_{\mathrm{N}_{2} \mathrm{D}^{+}}$, following the methods described above). The model curve (black) is shown in Figure 11. It has a velocity dispersion at core surface of $\sigma_{s}=0.306 \mathrm{~km} \mathrm{~s}^{-1}$. We also try a shallower density profile with $k_{\rho}=0.3$. This time we allow $k_{\sigma}$ to vary, finding a best-fit result $k_{\sigma}=-0.201$ and $\sigma_{s}=0.245 \mathrm{~km} \mathrm{~s}^{-1}$.

These results indicate that velocity dispersion is fairly constant with radius in the $\mathrm{C} 1-\mathrm{S}$ core. The fiducial turbulent core model is consistent with the profile of observed velocity dispersion versus projected radius, although its continuum flux profile is too concentrated (unless one invokes strong temperature and/or opacity gradients). A flatter density profile, i.e., $k_{\rho}=0.3$, which is more favored by the continuum flux profile, still implies a relatively flat velocity dispersion profile, although now declining with radius. However, these results 


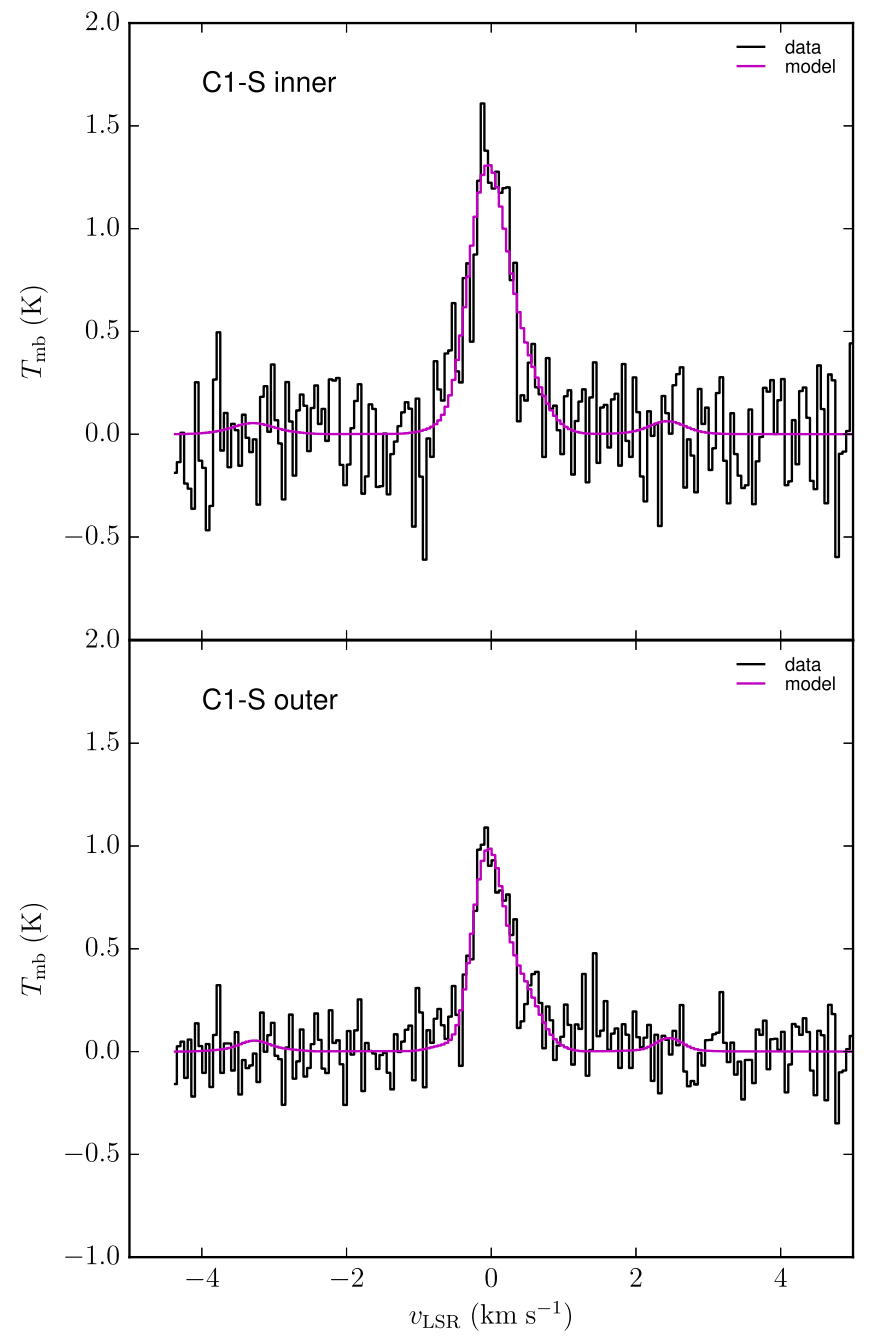

Figure 10. $\mathrm{N}_{2} \mathrm{D}^{+}(3-2)$ core spectra (black) and their HFS fit results (magenta). All spectra are in the rest frame of $79.4 \mathrm{~km} \mathrm{~s}^{-1}$. The spectral resolution is $0.05 \mathrm{~km} \mathrm{~s}^{-1}$.

assume a constant abundance of $\mathrm{N}_{2} \mathrm{D}^{+}$in the core, which is a caveat that needs to be borne in mind.

\subsubsection{Core Dynamics}

We follow T13 to measure the C1-S core properties at both the inner and outer scales and investigate its dynamics. We first use the MIREX image from Butler et al. (2014) to estimate the mass surface density of the core $\left(\Sigma_{c}\right)$ and its clump envelope $\left(\Sigma_{\mathrm{cl}, \mathrm{MIREX}}\right)$, evaluated from an annulus extending out to twice the core radius. However, we note that the C1-S region may be "saturated" in the MIREX map, i.e., it may provide only a lower limit to the actual mass surface density (see discussion in Butler \& Tan 2012; Butler et al. 2014; Lim \& Tan 2014). For the C1-S core we find $\Sigma_{\mathrm{c}, \text { MIREX }} \simeq 0.5 \mathrm{~g} \mathrm{~cm}^{-2}$ for both the inner and outer core regions. We also find quite similar values for the mass surface density of the clump via this method, i.e., $\Sigma_{\mathrm{cl}, \mathrm{MIREX}}$. This indicates that saturation in the map is indeed a problem. Another potential difficulty with estimates based on the MIREX map is that its angular resolution is only $2^{\prime \prime}$.

As a result of these potential problems with the MIREX map of this region, we also measure mass surface densities of the core and clump envelopes based on their millimeter continuum emission, i.e., $\Sigma_{\mathrm{c}, \mathrm{mm} \text {,tot }}$ and $\Sigma_{\mathrm{cl}, \mathrm{mm}}$. The subscript "tot" means
Table 3

Physical Properties of C1-S

\begin{tabular}{|c|c|c|}
\hline Core Property (\% Error) & C1-S Inner & C1-S Outer \\
\hline Angular radius, $\theta_{c}(\operatorname{arcsec})$ & 1.10 & 1.85 \\
\hline$d(\mathrm{kpc})(20 \%)$ & 5.0 & 5.0 \\
\hline$R_{\mathrm{c}}(0.01 \mathrm{pc})(20 \%)$ & 2.67 & 4.48 \\
\hline$V_{\mathrm{LSR}, \mathrm{N}_{2} \mathrm{D}^{+}}\left(\mathrm{km} \mathrm{s}^{-1}\right)$ & $79.30 \pm 0.0200$ & $79.30 \pm 0.0200$ \\
\hline$\sigma_{\mathrm{N}_{2} \mathrm{D}^{+}, \text {obs }}\left(\mathrm{km} \mathrm{s}^{-1}\right)$ & $0.242 \pm 0.0251$ & $0.212 \pm 0.0179$ \\
\hline$\sigma_{\mathrm{N}_{2} \mathrm{D}^{+}, \mathrm{nt}}\left(\mathrm{km} \mathrm{s}^{-1}\right)$ & $0.236 \pm 0.0257$ & $0.205 \pm 0.0185$ \\
\hline$\sigma_{\mathrm{N}_{2} \mathrm{D}^{+}}\left(\mathrm{km} \mathrm{s}^{-1}\right)$ & $0.302 \pm 0.0267$ & $0.279 \pm 0.0231$ \\
\hline$\Sigma_{\mathrm{cl}, \mathrm{MIREX}}\left(\mathrm{g} \mathrm{cm}^{-2}\right)(30 \%)$ & 0.490 & 0.445 \\
\hline$S_{\mathrm{cl}, \mathrm{mm}}(\mathrm{mJy})$ & $17.5 \pm 0.577$ & $23.8 \pm 1.04$ \\
\hline$S_{\mathrm{cl}, \mathrm{mm}} / \Omega\left(\mathrm{MJy} \mathrm{sr}^{-1}\right)$ & $78.7 \pm 2.60$ & $33.1 \pm 1.44$ \\
\hline$\Sigma_{\mathrm{cl}, \mathrm{mm}}\left(\mathrm{g} \mathrm{cm}^{-2}\right)$ & $0.798_{0.541}^{1.48}$ & $0.336_{0.228}^{0.624}$ \\
\hline$\Sigma_{\mathrm{c}, \operatorname{MIREX}}\left(\mathrm{g} \mathrm{cm}^{-2}\right)(30 \%)$ & 0.492 & 0.491 \\
\hline$M_{\mathrm{c}, \operatorname{MIREX}}\left(M_{\odot}\right)(50 \%)$ & 5.22 & 14.7 \\
\hline$n_{\mathrm{H}, \mathrm{c}, \mathrm{MIREX}}\left(10^{5} \mathrm{~cm}^{-3}\right)(36 \%)$ & 19.0 & 11.3 \\
\hline$S_{\mathrm{c}, \mathrm{mm}, \mathrm{tot}}(\mathrm{mJy})$ & $11.4 \pm 0.365$ & $23.2 \pm 0.577$ \\
\hline$S_{\mathrm{c}, \mathrm{mm}, \mathrm{tot}} / \Omega\left(\mathrm{MJy} \mathrm{sr}^{-1}\right)$ & $128 \pm 4.10$ & $104 \pm 2.60$ \\
\hline$\Sigma_{\mathrm{c}, \mathrm{mm}, \mathrm{tot}}\left(\mathrm{g} \mathrm{cm}^{-2}\right)$ & $2.41_{1.43}^{4.83}$ & $1.96_{1.16}^{3.93}$ \\
\hline$M_{\mathrm{c}, \mathrm{mm}, \mathrm{tot}}\left(M_{\odot}\right)$ & $25.5_{11.6}^{53.5}$ & $58.8_{26.8}^{123}$ \\
\hline$n_{\mathrm{H}, \mathrm{c}, \mathrm{mm}, \mathrm{tot}}\left(10^{5} \mathrm{~cm}^{-3}\right)$ & $93.0_{51.6}^{190}$ & $45.1_{25.0}^{92.1}$ \\
\hline$\Sigma_{\mathrm{c}, \mathrm{mm}}\left(\mathrm{g} \mathrm{cm}^{-2}\right)$ & $1.61_{0.628}^{4.03}$ & $1.63_{0.826}^{3.60}$ \\
\hline$M_{\mathrm{c}, \mathrm{mm}}\left(M_{\odot}\right)$ & $17.1_{4.97}^{43.7}$ & $48.8_{19.0}^{112}$ \\
\hline$n_{\mathrm{H}, \mathrm{c}, \mathrm{mm}}\left(10^{6} \mathrm{~cm}^{-3}\right)$ & $6.22_{2.24}^{15.8}$ & $3.73_{1.77}^{8.39}$ \\
\hline
\end{tabular}

Note. The superscripts (low temperature) and subscripts (high temperature) indicate the variations resulting from uncertainties in dust temperature (7 to $13 \mathrm{~K}$ for core; 10 to $20 \mathrm{~K}$ for clump).

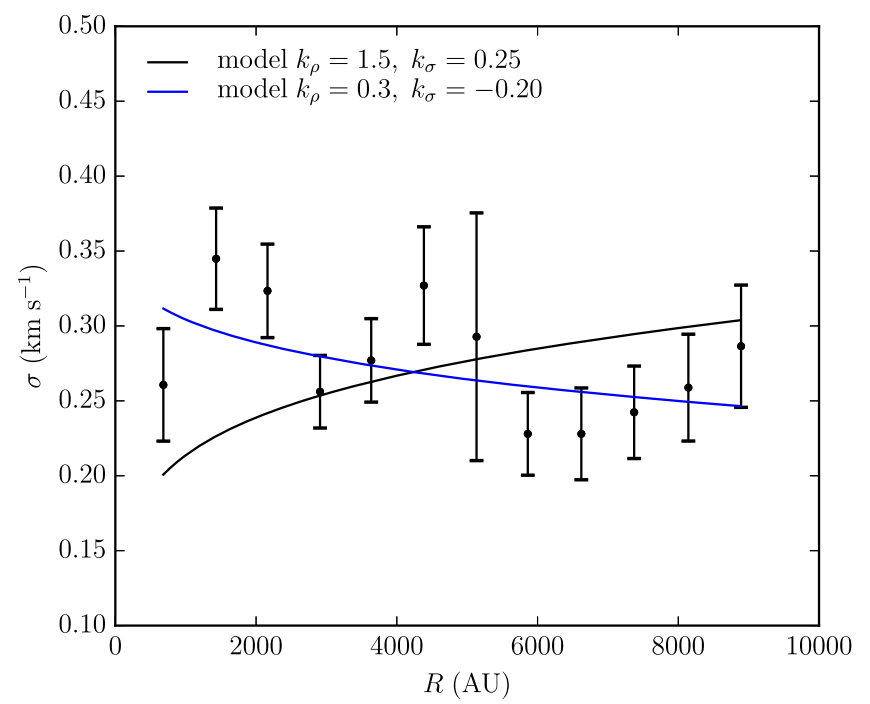

Figure 11. Velocity dispersion $\sigma$ as a function of projected radius, $R$, for C1-S outer. The observational measurement is averaged within concentric annuli at a step of beam size. Within each annulus, the observed $\sigma_{\mathrm{N}_{2} \mathrm{D}^{+}}$is derived following Section 3.2.3. The black model curve shows the $2 \mathrm{D}$ projection (mass-weighted sum in quadrature) of $\sigma(r) \propto r^{k_{\sigma}}\left(k_{\sigma}=0.25\right)$ with the density profile $\rho(r) \propto r^{-k_{\rho}}\left(k_{\rho}=1.5\right)$, following the fiducial MT03 model. The profile is normalized based on a $\chi^{2}$ minimization to the observation so that $\sigma$ at the core surface $(9250 \mathrm{au})$ is $\sigma_{\mathrm{s}}=0.306 \mathrm{~km} \mathrm{~s}^{-1}$. The blue model curve shows the model with a shallower density profile $\rho(r) \propto r^{-0.3}$ and the best-fit $k_{\sigma}=-0.201$. In this case, $\sigma_{s}=0.245 \mathrm{~km} \mathrm{~s}^{-1}$. 
no envelope subtraction (see below). Following T13, we adopt $T=10 \pm 3 \mathrm{~K}$ for the core, which results in lower and upper limits denoted by sub- and superscripts. For the clump we adopt $T=15 \pm 5 \mathrm{~K}$, i.e., with such temperatures being expected to be relevant for larger-scale regions in IRDCs (e.g., Pillai et al. 2006, see also Figure 2). With these methods we find that the $\mathrm{C} 1-\mathrm{S}$ core has $\Sigma_{\mathrm{c}, \mathrm{mm} \text {,tot }} \simeq 2.41$ and $1.96 \mathrm{~g} \mathrm{~cm}^{-2}$ at its inner and outer scales, respectively. The clump envelopes surrounding these apertures have $\Sigma_{\mathrm{cl}, \mathrm{mm}} \simeq$ 0.80 and $0.34 \mathrm{~g} \mathrm{~cm}^{-2}$, respectively. Given these results, we focus our attention on core properties for the dynamical analysis using the mass surface densities derived from the millimeter continuum.

We consider two cases for estimating core physical properties: (1) no envelope subtraction, i.e., using total millimeter continuum fluxes, for which we use the subscript "tot," e.g., $S_{\mathrm{c}, \mathrm{mm}, \mathrm{tot}}, \Sigma_{\mathrm{c}, \mathrm{mm}, \mathrm{tot}}, M_{\mathrm{c}, \mathrm{mm} \text {,tot }}$, etc.; (2) with envelope subtraction, for which we use variable names with no additional subscript, e.g., $\Sigma_{\mathrm{c}, \mathrm{mm}}, M_{\mathrm{c}, \mathrm{mm}}$, etc. The resulting physical properties of $\mathrm{C} 1-\mathrm{S}$, including volume densities (assuming spherical geometry and adopting a mean molecular weight of $2.33 m_{\mathrm{H}}$ ), are listed in Table 3. With the fiducial estimates of dust temperature, the derived masses of C1-S inner are $M_{\mathrm{c}, \mathrm{mm} \text {,tot }}=$ $25.5 M_{\odot}$ and $M_{\mathrm{c}, \mathrm{mm}}=17.1 M_{\odot}$, i.e., background subtraction makes a substantial difference in the mass estimate. For C1-S outer, the masses are $M_{\mathrm{c}, \mathrm{mm} \text {,tot }}=58.8 M_{\odot}$ and $M_{\mathrm{c}, \mathrm{mm}}=$ $48.8 M_{\odot}$, i.e., the effects of background subtraction are more modest (in a relative sense). The implied volume densities in $\mathrm{C} 1-\mathrm{S}$, also listed in Table 3 , are $n_{\mathrm{H}} \sim$ several $\times 10^{6} \mathrm{~cm}^{-3}$.

Now with estimates of the radii, masses, and velocity dispersions of the $\mathrm{C} 1-\mathrm{S}$ inner and outer core structures, along with the mass surface densities of their clump envelopes, we are able to compare these to predictions of the turbulent core model of MT03. Our general methodological approach follows that of T13. The mass-weighted average velocity dispersion of a virialized core, including pressure equilibrium with its surroundings, is given in the fiducial case by (MT03; T13)

$$
\sigma_{\mathrm{c}, \mathrm{vir}} \rightarrow 1.09\left(\frac{\phi_{B}}{2.8}\right)^{-3 / 8}\left(\frac{M_{c}}{60 M_{\odot}}\right)^{1 / 4}\left(\frac{\Sigma_{\mathrm{cl}}}{1 \mathrm{~g} \mathrm{~cm}^{-2}}\right)^{1 / 4} \mathrm{~km} \mathrm{~s}^{-1} \text {, }
$$

where $\phi_{B}=1.3+1.5 m_{\mathrm{A}}^{-2} \rightarrow 2.8$ is a dimensionless parameter that accounts for the effects of magnetic fields, $m_{\mathrm{A}} \equiv \sqrt{3} \sigma / v_{\mathrm{A}} \rightarrow 1$ is the Alfvén Mach number and $v_{\mathrm{A}}=B / \sqrt{4 \pi \rho}$ is the Alfvén speed. The fiducial case considered by MT03 (indicated by the $\rightarrow$ values, above) involved approximately equal support in the core by turbulence and large-scale magnetic fields. If magnetic fields play a more important role, then this is represented by a smaller Alfvén Mach number, i.e., sub-Alfvénic turbulence, and a larger value of $\phi_{B}$. In this case, a core in virial equilibrium would have a smaller turbulent line width.

Thus our basic procedure is to evaluate $\sigma_{\mathrm{c}, \mathrm{vir}}$ using Equation (2) and compare it with the observed 1D velocity dispersion inferred from the $\mathrm{N}_{2} \mathrm{D}^{+}(3-2)$ line, $\sigma_{\mathrm{N}_{2} \mathrm{D}^{+}}$. We do this for the $\mathrm{C} 1-\mathrm{S}$ inner and outer scales and for the cases with and without background subtraction of the clump envelope. These results are listed in Table 4 . The ratio $\sigma_{\mathrm{N}_{2} \mathrm{D}^{+}} / \sigma_{\mathrm{c} \text {,vir,mm }}$ is found to be approximately equal to 0.4 for both C1-S inner and outer, which is similar to the results of T13 based on the
Table 4

Dynamical Properties of C1-S

\begin{tabular}{|c|c|c|}
\hline Core Property & C1-S Inner & C1-S Outer \\
\hline $\begin{array}{l}R_{\mathrm{c}}(0.01 \mathrm{pc}) \\
\sigma_{\mathrm{N}_{2} \mathrm{D}^{+}}\left(\mathrm{km} \mathrm{s}^{-1}\right) \\
\Sigma_{\mathrm{cl}, \mathrm{mm}}\left(\mathrm{g} \mathrm{cm}^{-2}\right)\end{array}$ & $\begin{array}{c}2.67 \\
0.302 \pm 0.0267 \\
0.798_{0.541}^{1.48}\end{array}$ & $\begin{array}{c}4.48 \\
0.279 \pm 0.0231 \\
0.336_{0.228}^{0.624}\end{array}$ \\
\hline $\begin{array}{l}M_{\mathrm{c}, \mathrm{mm}, \mathrm{tot}}\left(M_{\odot}\right) \\
M_{\mathrm{c}, \mathrm{mm}}\left(M_{\odot}\right)\end{array}$ & $\begin{array}{l}25.5_{11.6}^{53.5} \\
17.1_{4.97}^{43.7}\end{array}$ & $\begin{array}{l}58.8_{26.8}^{123} \\
48.8_{19.0}^{112}\end{array}$ \\
\hline $\begin{array}{l}\sigma_{\mathrm{c}, \mathrm{vir}, \mathrm{mm}, \mathrm{tot}}\left(\mathrm{km} \mathrm{s}^{-1}\right) \\
\sigma_{\mathrm{c}, \mathrm{vir}, \mathrm{mm}}\left(\mathrm{km} \mathrm{s}^{-1}\right)\end{array}$ & $\begin{array}{l}0.832_{0.606}^{1.03} \\
0.752_{0.500}^{0.973}\end{array}$ & $\begin{array}{l}0.826_{0.602}^{1.02} \\
0.788_{0.557}^{0.994}\end{array}$ \\
\hline $\begin{array}{l}\sigma_{\mathrm{N}_{2} \mathrm{D}^{+}} / \sigma_{\mathrm{c}, \text { vir,mm,tot }} \\
\sigma_{\mathrm{N}_{2} \mathrm{D}^{+}} / \sigma_{\mathrm{c}, \mathrm{vir}, \mathrm{mm}}\end{array}$ & $\begin{array}{l}0.363_{0.293}^{0.499} \\
0.402_{0.311}^{0.604}\end{array}$ & $\begin{array}{l}0.337_{0.272}^{0.463} \\
0.354_{0.280}^{0.500}\end{array}$ \\
\hline $\begin{array}{l}n_{\mathrm{H}, \mathrm{c}, \mathrm{mm}, \mathrm{tot}}\left(10^{5} \mathrm{~cm}^{-3}\right) \\
n_{\mathrm{H}, \mathrm{c}, \mathrm{mm}}\left(10^{5} \mathrm{~cm}^{-3}\right)\end{array}$ & $\begin{array}{l}93.0_{51.6}^{190} \\
62.2_{22.4}^{158}\end{array}$ & $\begin{array}{l}45.1_{25.0}^{92.1} \\
37.3_{17.7}^{83.9}\end{array}$ \\
\hline $\begin{array}{l}t_{\mathrm{c}, \mathrm{ff}, \mathrm{tot}}\left(10^{5} \mathrm{yr}\right)^{\mathrm{a}} \\
t_{\mathrm{c}, \mathrm{ff}}\left(10^{5} \mathrm{yr}\right)^{\mathrm{a}}\end{array}$ & $\begin{array}{l}0.143_{0.100}^{0.192} \\
0.175_{0.110}^{0.291}\end{array}$ & $\begin{array}{l}0.206_{0.144}^{0.276} \\
0.226_{0.151}^{0.328}\end{array}$ \\
\hline $\begin{array}{l}\alpha_{\mathrm{c}, \text { tot }} \equiv 5 \sigma_{\mathrm{N}_{2} \mathrm{D}^{+}}^{2} R_{c} /\left(G M_{\mathrm{c}, \mathrm{mm}, \mathrm{tot}}\right)^{\mathrm{b}} \\
\alpha_{c} \equiv 5 \sigma_{\mathrm{N}_{2} \mathrm{D}^{+}}^{+} R_{c} /\left(G M_{\mathrm{c}, \mathrm{mm}}\right)^{\mathrm{b}}\end{array}$ & $\begin{array}{l}0.110_{0.0457}^{0.245} \\
0.165_{0.0554}^{0.568}\end{array}$ & $\begin{array}{l}0.0683_{0.0286}^{0.152} \\
0.0824_{0.0315}^{0.213}\end{array}$ \\
\hline $\begin{array}{l}B_{c, \text { tot }}(\mathrm{mG})\left(m_{\mathrm{A}}=1\right) \\
B_{c}(\mathrm{mG})\left(m_{\mathrm{A}}=1\right)\end{array}$ & $\begin{array}{l}0.865_{0.632}^{1.24} \\
0.708_{0.418}^{1.13}\end{array}$ & $\begin{array}{l}0.555_{0.406}^{0.798} \\
0.506_{0.343}^{0.761}\end{array}$ \\
\hline $\begin{array}{l}\phi_{\mathrm{B}, \mathrm{vir}, \mathrm{tot}} \\
\phi_{\mathrm{B}, \mathrm{vir}}\end{array}$ & $\begin{array}{l}41.733 .7 \\
31.9_{10.7}^{63.3}\end{array}$ & $\begin{array}{l}50.8_{21.8}^{89.8} \\
44.8_{17.8}^{83.2}\end{array}$ \\
\hline $\begin{array}{l}m_{\mathrm{A}, \mathrm{vir}, \text { tot }} \\
m_{\mathrm{A}, \mathrm{vir}}\end{array}$ & $\begin{array}{l}0.193_{0.144}^{0.301} \\
0.221_{0.156}^{0.399}\end{array}$ & $\begin{array}{l}0.174_{0.130}^{0.270} \\
0.186_{0.135}^{0.302}\end{array}$ \\
\hline $\begin{array}{l}B_{\mathrm{c}, \mathrm{vir}, \mathrm{tot}}(\mathrm{mG}) \\
B_{\mathrm{c}, \mathrm{vir}}(\mathrm{mG})\end{array}$ & $\begin{array}{l}4.49_{2.47}^{6.98} \\
3.19_{1.27}^{5.54}\end{array}$ & $\begin{array}{l}3.19_{1.77}^{4.95} \\
2.72_{1.36}^{4.43}\end{array}$ \\
\hline $\begin{array}{l}B_{\mathrm{c}, \text { crit,tot }}(\mathrm{mG}) \\
B_{\mathrm{c}, \mathrm{crit}}(\mathrm{mG})\end{array}$ & $\begin{array}{l}3.24_{1.95}^{5.42} \\
2.17_{0.873}^{4.19}\end{array}$ & $\begin{array}{l}2.64_{1.59}^{4.42} \\
2.19_{1.15}^{3.90}\end{array}$ \\
\hline
\end{tabular}

Notes. The superscripts (low temperature) and subscripts (high temperature) indicate the variations resulting from uncertainties in dust temperature (7 to $13 \mathrm{~K}$ for core; 10 to $20 \mathrm{~K}$ for clump).

${ }^{\text {a }}$ Core free-fall time, $t_{\mathrm{c}, \mathrm{ff}}=\left[3 \pi /\left(32 G \rho_{c}\right)\right]^{1 / 2}=1.38 \times 10^{5}\left(n_{\mathrm{H}, \mathrm{c}, \mathrm{mm}} / 10^{5} \mathrm{~cm}^{-3}\right)^{-1 / 2} \mathrm{yr}$. ${ }^{\mathrm{b}}$ Virial parameter (Bertoldi \& McKee 1992).

lower-resolution observations of C1-S and of Kong et al. (2017) for other $\mathrm{N}_{2} \mathrm{D}^{+}$cores in IRDCs.

There are two possible interpretations for these results. The first possibility is that the core really is in a sub-virial state, i.e., it is on the verge of undergoing fast collapse because it lacks sufficient internal pressure support. Evaluation of the virial parameter of Bertoldi \& McKee (1992) (see Table 4), which is often used to assess the dynamical state of cores, would also seem to indicate such a situation. However, the high densities of the core imply short free-fall times (also listed in Table 4), which are $\sim 2 \times 10^{4} \mathrm{yr}$. Gas in the core would be expected to relatively quickly acquire infall velocities, which would approach the free-fall speed and thus give the core an apparent velocity dispersion that is comparable to that of virial equilibrium. It seems unlikely that all the core material is slowly moving and on the verge of fast collapse, especially on the different scales of C1-S inner and outer. In addition, the astrochemical modeling of the deuteration process that increases the abundance of $\mathrm{N}_{2} \mathrm{D}^{+}$compared to $\mathrm{N}_{2} \mathrm{H}^{+}$is thought 
to take a relatively long time compared to the local free-fall time. The results of Kong et al. (2016) and Goodson et al. (2016) indicate that the C1-S core should be contracting at a rate smaller than $1 / 3$ of that of free-fall collapse in order to have enough time to reach the observed levels of deuteration.

The second possibility is that the $\mathrm{C} 1-\mathrm{S}$ core is quite close to a state of virial equilibrium and is only undergoing relatively slow contraction compared to that of free-fall collapse. This would then require stronger magnetic fields than those of the fiducial $\left(m_{\mathrm{A}}=1\right)$ turbulent core model. The values of these fiducial $B$-field strengths are about $0.5 \mathrm{mG}$ on the scale of $\mathrm{C} 1-\mathrm{S}$ outer and $0.8 \mathrm{mG}$ at $\mathrm{C} 1-\mathrm{S}$ inner. The values of $\phi_{B}$ to achieve virial equilibrium, $\phi_{\mathrm{B} \text {,vir }}$, are several times larger than that of the fiducial case (Table 4). These correspond to conditions of sub-Alfvénic turbulence with $m_{\mathrm{A}} \simeq 0.2$ and magnetic field strengths of $\sim 2$ to $3 \mathrm{mG}$. We note that such $B$-field strengths are consistent with the values predicted by the empirical relation for median values of Crutcher et al. (2010), $B_{\text {med }} \simeq 0.22\left(n_{\mathrm{H}} / 10^{5} \mathrm{~cm}^{-3}\right)^{0.65} \quad\left(\right.$ valid for $\left.n_{\mathrm{H}}>300 \mathrm{~cm}^{-3}\right)$, given the observed densities of $\mathrm{C} 1-\mathrm{S}$ inner and outer. This relation predicts $B$-field strengths of 3.2 and $2.7 \mathrm{mG}$ using the envelope-subtracted densities of $\mathrm{C} 1-\mathrm{S}$ inner and outer, respectively. Finally, such values of $B$-field strengths can also help to explain why $\mathrm{C} 1-\mathrm{S}$ does not appear to have fragmented significantly, i.e., based on its millimeter continuum morphology. ${ }^{9}$ Table 3 lists the magnetic field strengths, $B_{\mathrm{c}, \mathrm{crit}}$, that would be needed for the magnetic critical mass of Bertoldi \& McKee (1992) to equal the observed core masses: at the scale of C1-S outer we see that $B_{\mathrm{c} \text {, crit }} \simeq B_{\mathrm{c}, \mathrm{vir}} \simeq 2 \mathrm{mG}$.

In summary, given the above results, we conclude that the second case of core dynamics regulated by relatively strong, $\sim 2 \mathrm{mG}$, magnetic fields appears to be the more likely scenario. Such magnetic field strengths are reasonable given the observed densities. They help explain the fragmentation scale of $\mathrm{C} 1-\mathrm{S}$ outer, i.e., $\sim 50 M_{\odot}$. They would allow $\mathrm{C} 1-\mathrm{S}$ outer to be virialized and thus potentially relatively old compared to its free-fall time, which helps to explain its observed high level of deuteration of $\mathrm{N}_{2} \mathrm{H}^{+}$, i.e., high abundance of $\mathrm{N}_{2} \mathrm{D}^{+}$. We return to this point in Section 4, where we consider the implications of the observed $\mathrm{C} 1-\mathrm{S}$ properties for astrochemical models. The predictions of there being dynamically important magnetic fields are: (1) strong Zeeman splitting of species, such as $\mathrm{CN}$, if they are present in the gas phase within the core; (2) ordered polarization angles for dust continuum emission, assuming that dust grains can align with the $B$-fields; (3) relatively small infall rates compared to free-fall. Again, we return to discussion of these predictions below in Section 4 .

\subsection{CO Outflows}

Figure 12 shows the new high-resolution $\mathrm{CO}(2-1)$ data, which can be compared to the compact-configuration results presented by T16. In the figure we show both the extendedonly data (Figure 12(a)), which emphasize the finest structures, and the combined data (Figure 12(b)). In panel (a), we can see a very narrow and collimated bipolar outflow launched from $\mathrm{C} 1$ $\mathrm{Sa}$. We draw a white line to roughly represent the outflow axis. We also draw a yellow line to show the approximate

\footnotetext{
9 Note the fluctuations of order unity in $\mathrm{N}_{2} \mathrm{D}^{+}$column density within $\mathrm{C} 1-\mathrm{S}$ are easily within the range expected from abundance variations (Goodson et al. 2016). Furthermore, we have carried out a similar dynamical analysis on local $\mathrm{N}_{2} \mathrm{D}^{+}$peaks and find these to be relatively more gravitationally stable than C1-S at its inner and outer scales.
}

orientation of the continuum elongation. The position angle between the two lines is about $60^{\circ}$, which means the angle between the normal direction to the continuum elongation and the outflow axis is approximately $30^{\circ}$. Also relevant is that the origin of the outflow appears to be slightly offset from the continuum peak (by one beam). The outflow is only marginally resolved in the direction perpendicular to the flow axis (by $\sim 3$ beams). An upper limit on the half-opening angle of the outflow cavity is estimated to be $\lesssim 10^{\circ}$. One can see clumpy structures in both the blue and red lobes. The redshifted outflow appears to change the orientation of its flow axis by a small angle, $\sim 10^{\circ}$, once it is about $0.1 \mathrm{pc}$ away from the protostar. The blueshifted side does not show evidence for such a deflection; however, its overlap with the C1-Sb outflow makes this harder to discern. The observed extent of the outflow appears to be set by sensitivity, rather than being a real, physical limit.

The outflow from $\mathrm{C} 1-\mathrm{Sb}$ shows a wider opening angle at its base. The cavity walls, i.e., outflow lobe boundaries, are apparent in the extended-configuration image. The blue lobe overlaps with that from $\mathrm{C} 1-\mathrm{Sa}$, but it is not clear whether this involves a physical interaction or is simply a projection effect.

There appear to be some high-velocity $\mathrm{CO}(2-1)$ emission features in the wider region. However, some of these features may be artifacts resulting from imperfect cleaning of the image, given the strong sources of the C1-Sa and C1-Sb outflows. Or these features could be outflows from other nearby protostars or other ambient high-velocity gas in the IRDC or projected along the line of sight.

Finally and most importantly for the purposes of this paper, neither panel (a) nor panel (b) of Figure 12 shows any hint of $\mathrm{CO}$ outflows from $\mathrm{C} 1-\mathrm{S}$. This is further evidence that the $\mathrm{C} 1-\mathrm{S}$ core is likely to be starless.

\subsection{Ancillary Molecular Lines}

Figure 13 shows the zeroth-moment maps of $\mathrm{C}^{18} \mathrm{O}(2-1)$, $\mathrm{DCO}^{+}(3-2)$, and $\mathrm{DCN}(3-2)$. The integrations are centered on $79.4 \mathrm{~km} \mathrm{~s}^{-1}$ and within a range from 76.9 to $81.9 \mathrm{~km} \mathrm{~s}^{-1}$. Note that in Figure 6 there is another velocity component at about $75 \mathrm{~km} \mathrm{~s}^{-1}$ seen in $\mathrm{C}^{18} \mathrm{O}(2-1)$, but this is not included in these maps. $\mathrm{C}^{18} \mathrm{O}(2-1)$ emission is nearly absent from $\mathrm{C} 1-\mathrm{S}$ (see also the spectra shown in Figure 6), but is rather mostly concentrated in $\mathrm{C} 1-\mathrm{Sa}$ and $\mathrm{C} 1-\mathrm{Sb}$.

The lack of $\mathrm{C}^{18} \mathrm{O}(2-1)$ emission in $\mathrm{C} 1-\mathrm{S}$ can be explained by $\mathrm{CO}$ depletion from the gas phase. In the $\mathrm{C} 1-\mathrm{S}$ core and much of its clump envelope, we expect that the temperature is $\lesssim 15 \mathrm{~K}$ (see, e.g., Figure 2). At such temperatures, CO is mostly frozen-out onto dust grains (Caselli \& Ceccarelli 2012). As shown in Figure 6(3c), $\mathrm{C} 1-\mathrm{S}$ inner has no detected $\mathrm{C}^{18} \mathrm{O}(2-1)$ flux (i.e., at velocities immediately around $79.4 \mathrm{~km} \mathrm{~s}^{-1}$ overlapping with the $\mathrm{N}_{2} \mathrm{D}^{+}(3-2)$ emission). We use the $3 \sigma$ zeroth-moment $\mathrm{C}^{18} \mathrm{O}(2-1)$ noise level $\left(0.446 \mathrm{~K} \mathrm{~km} \mathrm{~s}^{-1}\right.$ for $\mathrm{C} 1-\mathrm{S}$ inner) to calculate an upper limit of $\mathrm{C}^{18} \mathrm{O}$ column density $N_{\mathrm{C}^{8} \mathrm{O}}^{\mathrm{Cl}}$-Sinner $<3.00 \times 10^{14} \mathrm{~cm}^{-2}$, assuming an excitation temperature of $10 \mathrm{~K}$ and optically thin conditions. This corresponds to an abundance constraint of $\left[\mathrm{C}^{18} \mathrm{O}\right] /[\mathrm{H}]=$ $2.91 \times 10^{-10}$ (using $\Sigma_{\mathrm{c}, \mathrm{mm} \text {,tot }}$ from Table 3 and assuming that $1.0 \mathrm{~g} \mathrm{~cm}^{-2}$ corresponds to $N_{\mathrm{H}}=4.27 \times 10^{23} \mathrm{~cm}^{-2}$. Assuming $\left[{ }^{12} \mathrm{CO} / \mathrm{H}\right]=10^{-4}$ and $\left[{ }^{16} \mathrm{O} /{ }^{18} \mathrm{O}\right]=557$ (Wilson 1999), the above abundance constraint in $\mathrm{C} 1-\mathrm{S}$ inner corresponds to a $\mathrm{CO}$ depletion factor of $f_{D}>616$. Such high CO depletion factors have also been found toward the center of the lower-mass pre-stellar 
(a)

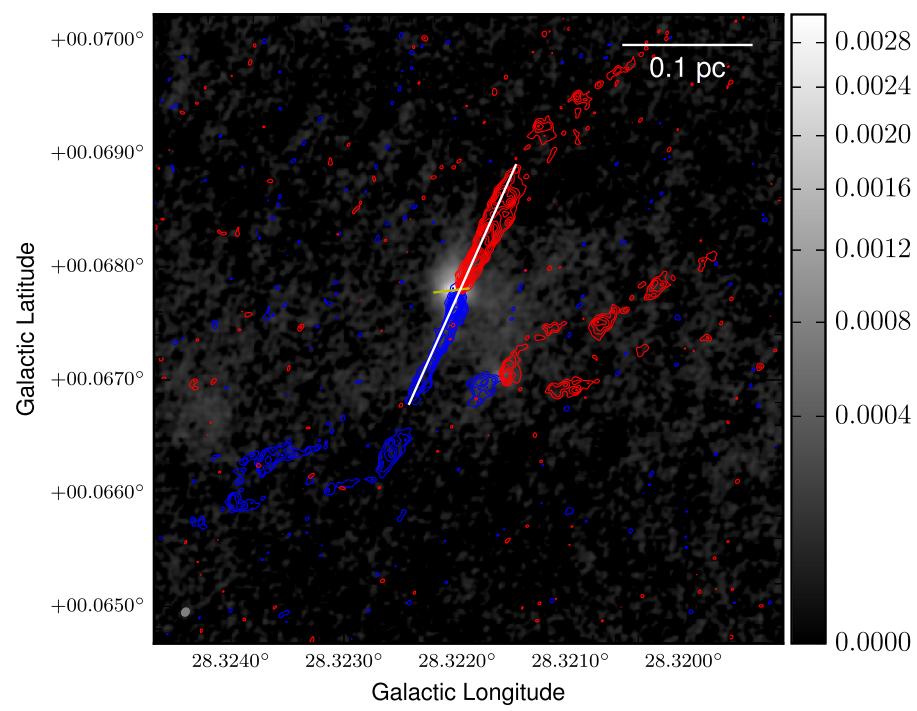

(b)

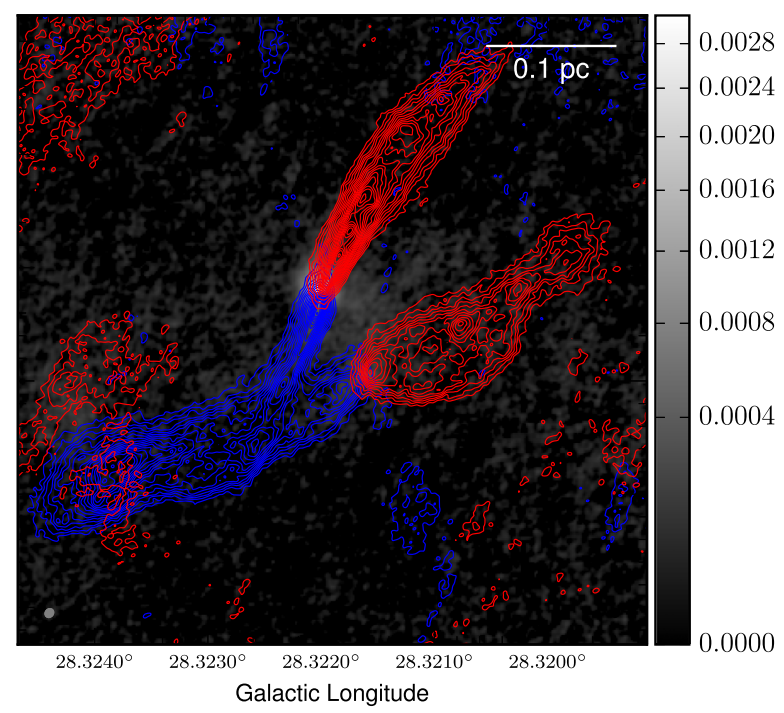

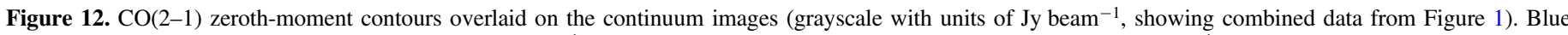

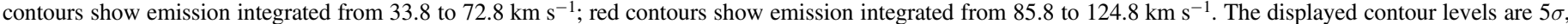

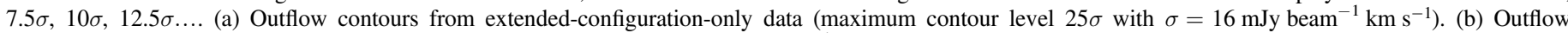

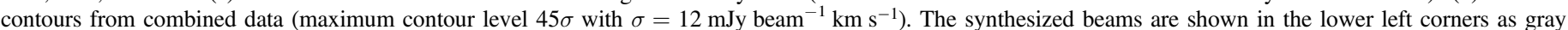
filled ellipses.
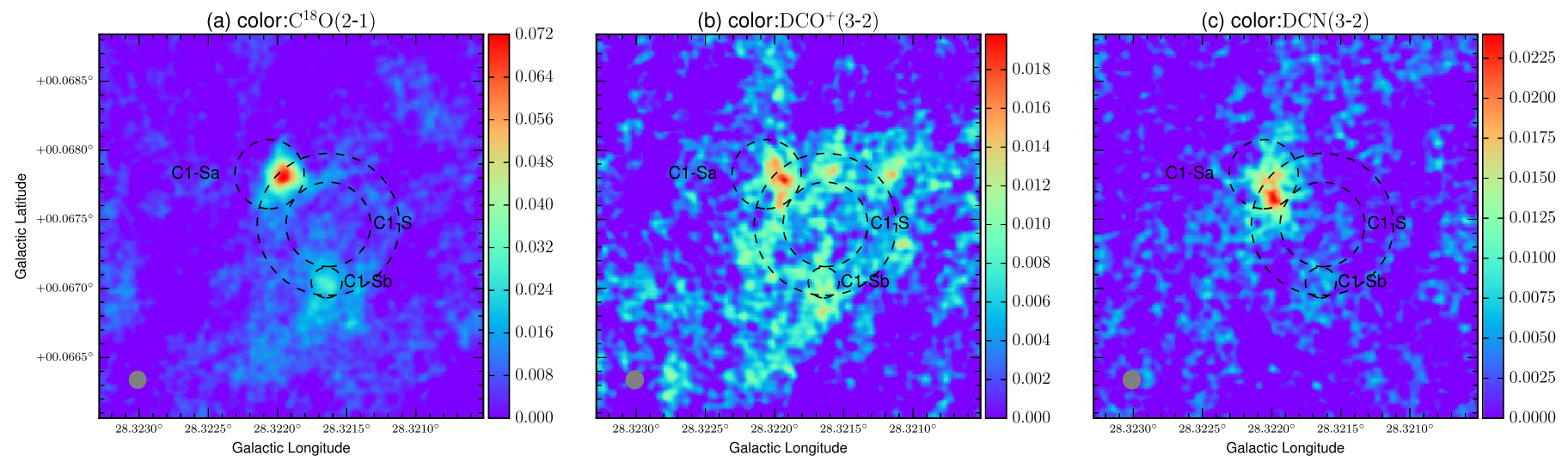

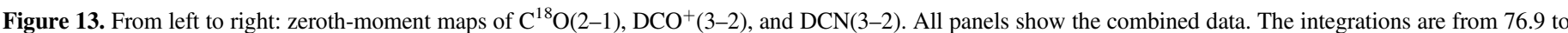
$81.9 \mathrm{~km} \mathrm{~s}^{-1}$. The color scale bars have units of Jy beam ${ }^{-1} \mathrm{~km} \mathrm{~s}^{-1}$. The synthesized beams $(\sim 0$ !" 3$)$ are shown as gray filled ellipses in the lower left corners.

core L1544 (Caselli et al. 1999, 2002), suggesting a similarity of astrochemical properties of this source with $\mathrm{C} 1-\mathrm{S}$.

$\mathrm{C}^{18} \mathrm{O}(2-1)$ emission is observed toward the protostars $\mathrm{C} 1-\mathrm{Sa}$ and $\mathrm{C} 1-\mathrm{Sb}$. This is to be expected, since in the protostellar models of Zhang et al. (2014) and Zhang \& Tan (2015), earlystage protostellar cores can have mean temperatures up to $T \sim 30 \mathrm{~K}$, which is significantly above the CO sublimation temperature of $17 \mathrm{~K}$ (van Dishoeck et al. 1993).

The zeroth-moment map of $\mathrm{DCO}^{+}(3-2)$ shows emission from $\mathrm{C} 1-\mathrm{Sa}$, a relative lack of emission from $\mathrm{C} 1-\mathrm{S}$ inner (consistent with the large amount of CO freeze-out), and hints of emission from the envelope around C1-S inner. DCN(3-2) shows strong emission only from the $\mathrm{C} 1-\mathrm{Sa}$ protostar, though with enhancement slightly offset from the peak of the continuum emission.

Figure 14 shows the first-moment maps of $\mathrm{C}^{18} \mathrm{O}(2-1)$, $\mathrm{DCO}^{+}(3-2)$, and $\mathrm{DCN}(3-2)$, evaluated using the same velocity range as the zeroth-moment maps shown in Figure 13. Only cells above $3 \sigma$ values are included. The $\mathrm{C}^{18} \mathrm{O}(2-1)$ firstmoment map clearly shows two velocity components. Much of the region is filled with the $81.2 \mathrm{~km} \mathrm{~s}^{-1}$ component, while in $\mathrm{C} 1-\mathrm{Sa}$ the $79.4 \mathrm{~km} \mathrm{~s}^{-1}$ component dominates.

A plausible overall picture of the $\mathrm{C} 1$ region is the following. There are two overlapping clouds, one at $79.4 \mathrm{~km} \mathrm{~s}^{-1}$ and the other at $81.2 \mathrm{~km} \mathrm{~s}^{-1}$. The first one has strong $\mathrm{CO}$ depletion (except in a local region around the protostar $\mathrm{C} 1-\mathrm{Sa}$ ) and deuteration (especially with $\mathrm{N}_{2} \mathrm{D}^{+}$), while the latter still has abundant $\mathrm{CO}$ but little $\mathrm{N}_{2} \mathrm{D}^{+}$. C1-Sa and $\mathrm{C} 1-\mathrm{S}$ are in the $79.4 \mathrm{~km} \mathrm{~s}^{-1}$ cloud, while $\mathrm{C} 1-\mathrm{Sb}$ is in the $81.2 \mathrm{~km} \mathrm{~s}^{-1}$ cloud. While $\mathrm{C} 1-\mathrm{S}$ shows a narrow line width in $\mathrm{N}_{2} \mathrm{D}^{+}(3-2), \mathrm{C} 1-\mathrm{Sa}$ line profiles are broader (i.e., dispersion of $0.32 \mathrm{~km} \mathrm{~s}^{-1}$ from an HFS fit, 1.3 times larger than that of $\mathrm{C} 1-\mathrm{S}$ inner), perhaps being caused by its outflows (Figure 6). It is unclear whether the $79.4 \mathrm{~km} \mathrm{~s}^{-1}$ and $81.2 \mathrm{~km} \mathrm{~s}^{-1}$ clouds are interacting or not. 

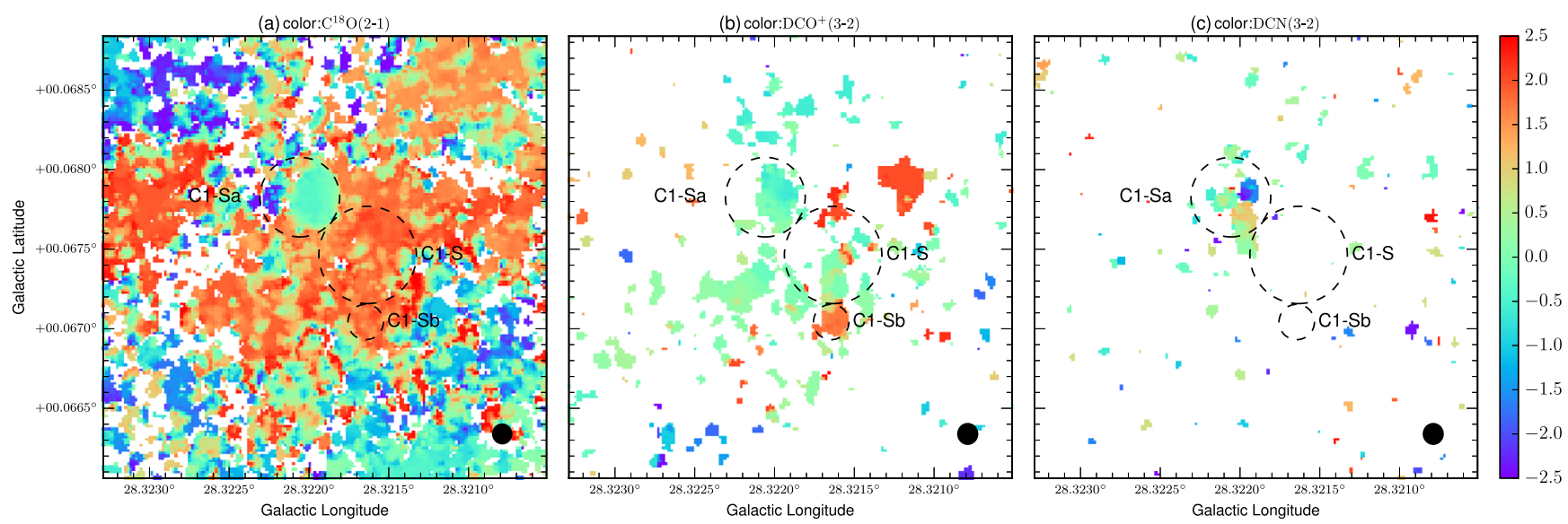

Figure 14. From left to right: first-moment maps of $\mathrm{C}^{18} \mathrm{O}(2-1), \mathrm{DCO}^{+}(3-2)$, and $\mathrm{DCN}(3-2)$. The integration is over $79.4 \pm 2.5 \mathrm{~km} \mathrm{~s}{ }^{-1}$. Only cells above $3 \sigma$ are considered. The first moment is then normalized to the rest frame of $79.4 \mathrm{~km} \mathrm{~s}^{-1}$. The beams are shown as the black filled ellipses in the lower right corners. The color bar has units of $\mathrm{km} \mathrm{s}^{-1}$.

\subsection{Astrochemical Modeling of C1-S}

We carry out an astrochemical model of C1-S inner given its observed properties. The chemical model is from Kong et al. (2015): it follows the time-dependent change of more than 100 species, including $\mathrm{N}_{2} \mathrm{D}^{+}$. We adopt $n_{\mathrm{H}}=6 \times 10^{6} \mathrm{~cm}^{-3}$, i.e., the envelope-subtracted estimate based on millimeter continuum emission. We set gas and dust temperatures to $10 \mathrm{~K}$ and the heavy element depletion factor to 600 (based on estimation of $\mathrm{C}^{18} \mathrm{O}$ abundance in Section 3.4). Note that we are assuming this depletion factor for the elemental abundances of carbon, oxygen, and nitrogen. The model results show that the equilibrium abundance of $\mathrm{N}_{2} \mathrm{D}^{+}$is $\left[\mathrm{N}_{2} \mathrm{D}^{+}\right] /[\mathrm{H}]=1.94 \times$ $10^{-11}$. If we decrease/increase $n_{\mathrm{H}}$ by a factor of 2 , the equilibrium value of $\left[\mathrm{N}_{2} \mathrm{D}^{+}\right] /[\mathrm{H}]=(1.84,1.90) \times 10^{-11}$, respectively. If we set $T=(7,13) \mathrm{K}$, the equilibrium $\left[\mathrm{N}_{2} \mathrm{D}^{+}\right] /[\mathrm{H}]=\left(\begin{array}{ll}1.70, & 2.10\end{array}\right) \times 10^{-11}$, respectively. If we decrease/increase $f_{D}$ by a factor of 2 , the equilibrium $\left[\mathrm{N}_{2} \mathrm{D}^{+}\right] /[\mathrm{H}]=(3.11,0.94) \times 10^{-11}$, respectively. If we decrease/increase the cosmic ionization rate by a factor of 2 , the equilibrium $\left[\mathrm{N}_{2} \mathrm{D}^{+}\right] /[\mathrm{H}]=(1.91,1.84) \times 10^{-11}$, respectively. These results illustrate the sensitivity of the theoretical equilibrium abundance of $\mathrm{N}_{2} \mathrm{D}^{+}$to astrochemical model parameters.

On the observational side, if we assume an $\mathrm{N}_{2} \mathrm{D}^{+}(3-2)$ excitation temperature $T_{\mathrm{ex}}=10 \mathrm{~K}$, the $\mathrm{C} 1-\mathrm{S}$ inner $\mathrm{N}_{2} \mathrm{D}^{+}$column density is $1.39 \times 10^{12} \mathrm{~cm}^{-2}$. Using $\Sigma_{\mathrm{c}, \mathrm{mm}}=1.61 \mathrm{~g} \mathrm{~cm}^{-2}$ from Table 3, we derive an $\mathrm{N}_{2} \mathrm{D}^{+}$abundance $\left[\mathrm{N}_{2} \mathrm{D}^{+}\right] /[\mathrm{H}]=2.02 \times$ $10^{-12}$. Considering the possibility of subthermal excitation, we also try $T_{\mathrm{ex}}=6.4 \mathrm{~K}$ (Fontani et al. 2011), which yields $\left[\mathrm{N}_{2} \mathrm{D}^{+}\right] /[\mathrm{H}]=4.90 \times 10^{-12}$. For $T_{\mathrm{ex}}=4.1 \mathrm{~K}$, which was the low end of the range considered by Kong et al. (2016), $\left[\mathrm{N}_{2} \mathrm{D}^{+}\right] /[\mathrm{H}]=2.82 \times 10^{-11}$.

Given these results, we conclude that if $T_{\mathrm{ex}} \simeq 4 \mathrm{~K}$, then the observed abundance of $\mathrm{N}_{2} \mathrm{D}^{+}$in $\mathrm{C} 1-\mathrm{S}$ inner is consistent with the equilibrium value predicted by our astrochemical model for the observed density, temperature, and depletion factor. The timescale to reach $90 \%$ of the equilibrium is $1.36 \times 10^{5} \mathrm{yr}$ for an initial ortho-to-para ratio of $\mathrm{H}_{2}$ of 3 . This is about a factor of 8 longer than the free-fall time of $\mathrm{C} 1-\mathrm{S}$ inner. If the initial ortho-to-para ratio of $\mathrm{H}_{2}$ is 0.1 , the timescale will be shortened by a factor of 1.3 (Kong et al. 2015).

\section{Discussion and Conclusions}

We have presented follow-up observations at higher angular resolution of the C1-S core, first identified by its $\mathrm{N}_{2} \mathrm{D}^{+}(3-2)$ emission by T13. The CO observations of T16 identified two protostellar outflow sources near $\mathrm{C} 1-\mathrm{S}$ and it was speculated that one of these, C1-Sa, may be forming from the core. However, now with about $10 \times$ better angular resolution we are able to see that $\mathrm{C} 1-\mathrm{S}$, as traced by its $\mathrm{N}_{2} \mathrm{D}^{+}(3-2)$ emission, is distinct from the $\mathrm{C} 1-\mathrm{Sa}$ and $\mathrm{C} 1-\mathrm{Sb}$ protostars. Thus $\mathrm{C} 1-\mathrm{S}$ remains a good candidate to be a massive $\left(\sim 50 M_{\odot}\right)$ starless core, being highly deuterated and CO-depleted $\left(f_{D} \gtrsim 600\right)$ and lacking concentrated millimeter continuum emission or any sign of CO outflows (see the summary Figure 15).

The velocity dispersion of C1-S, as traced by $\mathrm{N}_{2} \mathrm{D}^{+}(3-2)$, indicates either sub-virial conditions in the trans-Alfvénic case or that the core is in approximate virial equilibrium with stronger magnetic fields $(\sim 2 \mathrm{mG})$ that dominate over turbulent pressure support, i.e., turbulence is sub-Alfvénic within the core. We favor the latter interpretation, since such $B$-field strengths are consistent with an extrapolation of the $B$ versus density relation of Crutcher et al. (2010). They also help explain the mass of C1-S at its outer scale as being set by the magnetic critical mass. Astrochemical modeling indicates that a relatively long core age compared to the free-fall time is needed to reach the observed highly deuterated state, and once a core is older than a few free-fall times, it is expected to be able to approach approximate virial equilibrium. Predictions of this scenario are that the continuum emission from C1-S will show strong polarization and that Zeeman splitting may be observed from species such as $\mathrm{CN}$, if they remain in the gas phase at a level high enough to be observed. Unfortunately the weakness of the dust continuum emission and the apparent high heavy element depletion factor in C1-S may make these tests difficult to carry out. Another prediction is that infall velocities, i.e., as traced by inverse P-Cygni profiles from suitable species (e.g., the $\mathrm{N}_{2} \mathrm{H}^{+}(1-0)$ line, as has been observed in lower-mass prestellar cores; Keto \& Caselli 2010), will show infall speeds that are a small fraction of the local free-fall speed.

These observations break new ground in being able to resolve the kinematic structure of the massive starless core. We have been able to measure the radial profile of turbulence in the core, which is seen to have a relatively constant velocity dispersion with radius. We have presented high-resolution first-moment 
(a) $\mathrm{N}_{2} \mathrm{D}^{+}(3-2)$

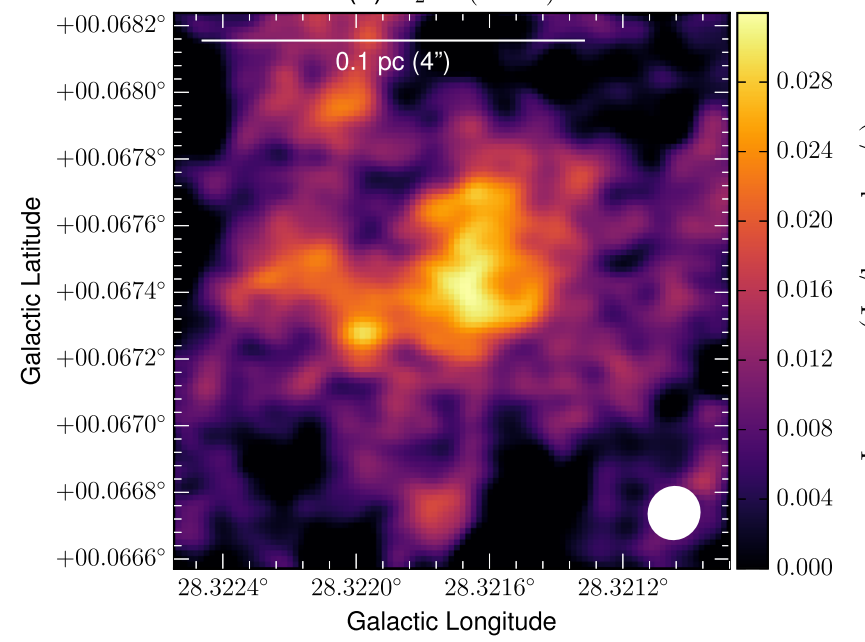

(b) $\mathrm{N}_{2} \mathrm{D}^{+}(3-2)+\mathrm{CO}(2-1)$

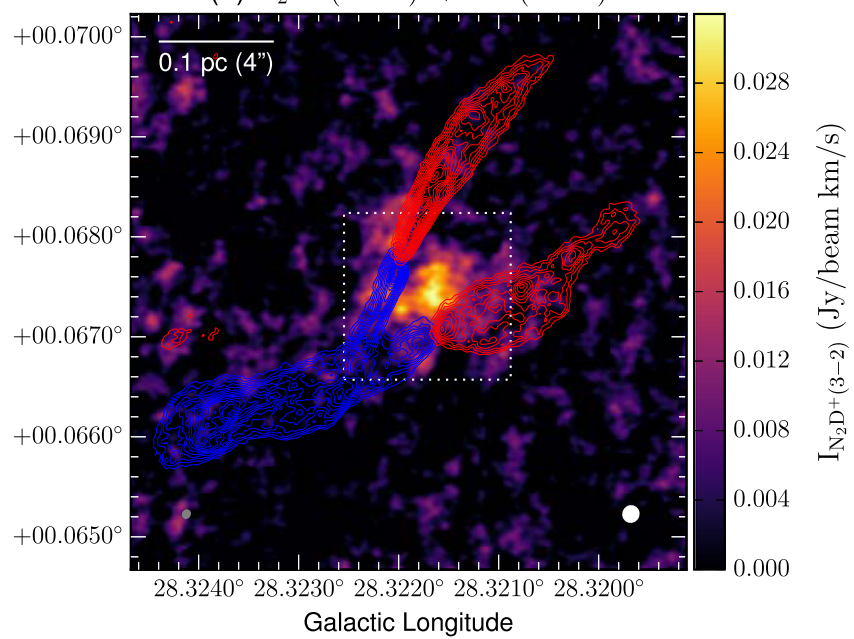

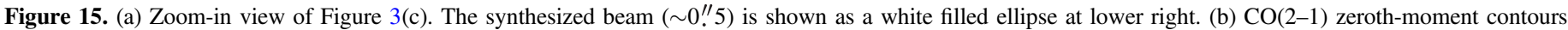

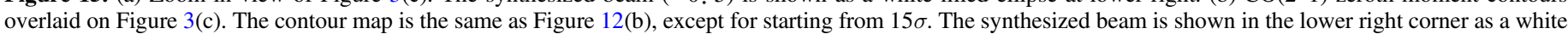
filled ellipse. The dotted square shows the zoom-in region of panel (a).

maps of the internal velocity structure. The statistics of these kinematic properties can be compared against numerical simulations of such cores (Goodson et al. 2016). We defer a detailed comparison with such simulations to a future paper.

The observations presented here have also revealed more details about the $\mathrm{C} 1-\mathrm{Sa}$ and $\mathrm{C} 1-\mathrm{Sb}$ protostars. $\mathrm{C} 1-\mathrm{Sa}$ is consistent with being a massive protostellar core $\left(\sim 30 M_{\odot}\right)$ that is in a very early stage of formation. It exhibits a very collimated outflow with a half-opening angle of $\lesssim 10^{\circ}$. The presence of $\mathrm{N}_{2} \mathrm{D}^{+}$in $\mathrm{C} 1-\mathrm{Sa}$ is consistent with the findings of Emprechtinger et al. (2009), who found that $D_{\text {frac }}^{\mathrm{N}_{2} \mathrm{H}^{+}}$can be as large as $20 \%$ in young low-mass protostars, while it declines in more evolved sources. C1-Sb appears to be a lower-mass protostellar core $\left(\sim 2 M_{\odot}\right)$, but at a later stage of development, e.g., with a wider opening angle of the outflow cavity.

The overall presence of a massive starless core alongside a relatively massive protostellar core and lower-mass protostellar core gives interesting clues about the early stages of massive star formation, at least in one case. The environment within this region of about $0.1 \mathrm{pc}$ projected scale is not especially crowded, particularly considering that $\mathrm{C} 1-\mathrm{Sb}$ seems to be at a quite different velocity and may be physically separated along the line of sight. This leaves just the C1-S and C1-Sa sources, both relatively massive, monolithic objects. There is no evidence so far for a cluster of protostars that all drive outflows, which is an expectation of competitive accretion models (e.g., Bonnell et al. 2001; Wang et al. 2010). The current observations can easily detect sources such as $\mathrm{C} 1-\mathrm{Sb}$ that have about a $2 M_{\odot}$ protostellar core and its level of protostellar outflow activity, if they are present. The $5 \sigma$ continuum mass sensitivity is $0.15 M_{\odot}$ per beam (for $T=$ $20 \mathrm{~K})$.

Deeper, more sensitive observations with ALMA will lead to improved understanding of these sources. For example, highersensitivity $\mathrm{N}_{2} \mathrm{D}^{+}$observations can better probe the kinematic substructure of the $\mathrm{C} 1-\mathrm{S}$ core. Other transitions should be observed to constrain the excitation temperature of this species. Similar observations of $\mathrm{N}_{2} \mathrm{H}^{+}$are needed to map the deuteration structure. Observations with higher sensitivity and angular resolution in high-velocity $\mathrm{CO}(2-1)$ and in the millimeter dust continuum will enable more stringent constraints to be placed on the protostellar content of this region. A search for centimeter continuum emission is needed to better constrain models (Tanaka et al. 2017) of the potentially massive protostar $\mathrm{C} 1-\mathrm{Sa}$.

We thank an anonymous referee for comments that improved the paper. We thank Mengyao Liu, Matthew Goodson, Crystal Brogan, Jim Braatz, Sarah Wood, Shawn Booth, and Héctor Arce for helpful discussions. S.K. was funded by NSF award AST1140063 while conducting this study. J.C.T. acknowledges an NRAO/SOS grant and NSF grant AST1411527. P.C. acknowledges the financial support of the European Research Council (ERC; project PALs 320620). This paper makes use of the following ALMA data: ADS/JAO.ALMA\#2013.1.00248.S. ALMA is a partnership of ESO (representing its member states), NSF (USA), and NINS (Japan), together with NRC (Canada), NSC and ASIAA (Taiwan), and KASI (Republic of Korea), in cooperation with the Republic of Chile. The Joint ALMA Observatory is operated by ESO, AUI/NRAO, and NAOJ. The National Radio Astronomy Observatory is a facility of the National Science Foundation operated under cooperative agreement by Associated Universities, Inc.

Facility: ALMA; VLA.

\section{ORCID iDs}

Shuo Kong (iD https://orcid.org/0000-0002-8469-2029

Jonathan C. Tan (10 https://orcid.org/0000-0002-3389-9142

Paola Caselli (iD https://orcid.org/0000-0003-1481-7911

Francesco Fontani (iD https://orcid.org/0000-0003-0348-3418

Ke Wang (i) https://orcid.org/0000-0002-7237-3856

Michael J. Butler (10) https://orcid.org/0000-0003-1031-898X

\section{References}

Bertoldi, F., \& McKee, C. F. 1992, ApJ, 395, 140

Bonnell, I. A., Bate, M. R., Clarke, C. J., \& Pringle, J. E. 2001, MNRAS, 323, 785

Bontemps, S., Motte, F., Csengeri, T., \& Schneider, N. 2010, A\&A, 524, A18 Butler, M. J., \& Tan, J. C. 2012, ApJ, 754, 5

Butler, M. J., Tan, J. C., \& Kainulainen, J. 2014, ApJL, 782, L30

Caselli, P., \& Ceccarelli, C. 2012, A\&ARv, 20, 56 
Caselli, P., Walmsley, C. M., Tafalla, M., Dore, L., \& Myers, P. C. 1999, ApJL, 523, L165

Caselli, P., Walmsley, C. M., Zucconi, A., et al. 2002, ApJ, 565, 344

Crapsi, A., Caselli, P., Walmsley, M. C., \& Tafalla, M. 2007, A\&A, 470, 221

Crutcher, R. M., Wandelt, B., Heiles, C., Falgarone, E., \& Troland, T. H. 2010, ApJ, 725, 466

Cyganowski, C. J., Brogan, C. L., Hunter, T. R., et al. 2014, ApJL, 796, L2

Duarte-Cabral, A., Bontemps, S., Motte, F., et al. 2013, A\&A, 558, A125

Emprechtinger, M., Caselli, P., Volgenau, N. H., Stutzki, J., \& Wiedner, M. C. 2009, A\&A, 493, 89

Feng, S., Beuther, H., Zhang, Q., et al. 2016, ApJ, 828, 100

Fontani, F., Palau, A., Caselli, P., et al. 2011, A\&A, 529, L7

Friesen, R. K., Di Francesco, J., Bourke, T. L., et al. 2014, ApJ, 797, 27

Goodson, M. D., Kong, S., Tan, J. C., Heitsch, F., \& Caselli, P. 2016, ApJ, 833, 274

Henshaw, J. D., Caselli, P., Fontani, F., et al. 2013, MNRAS, 428, 3425

Ho, P. T. P., \& Townes, C. H. 1983, ARA\&A, 21, 239

Keto, E., \& Caselli, P. 2010, MNRAS, 402, 1625

Kong, S., Caselli, P., Tan, J. C., Wakelam, V., \& Sipilä, O. 2015, ApJ, 804, 98

Kong, S., Tan, J. C., Caselli, P., et al. 2016, ApJ, 821, 94

Kong, S., Tan, J. C., Caselli, P., et al. 2017, ApJ, 834, 193

Lim, W., \& Tan, J. C. 2014, ApJL, 780, L29
Lim, W., Tan, J. C., Kainulainen, J., Ma, B., \& Butler, M. J. 2016, ApJL, 829, L19 McKee, C. F., \& Tan, J. C. 2002, Natur, 416, 59

McKee, C. F., \& Tan, J. C. 2003, ApJ, 585, 850

Ossenkopf, V., \& Henning, T. 1994, A\&A, 291, 943

Pillai, T., Wyrowski, F., Carey, S. J., \& Menten, K. M. 2006, A\&A, 450, 569

Rathborne, J. M., Jackson, J. M., \& Simon, R. 2006, ApJ, 641, 389

Rosolowsky, E. W., Pineda, J. E., Foster, J. B., et al. 2008, ApJS, 175, 509

Shu, F. H. 1977, ApJ, 214, 488

Tan, J. C., Beltrán, M. T., Caselli, P., et al. 2014, in Protostars and Planets VI, ed. H. Beuther et al. (Tucson, AZ: Univ. Arizona Press), 149

Tan, J. C., Kong, S., Butler, M. J., Caselli, P., \& Fontani, F. 2013, ApJ, 779, 96

Tan, J. C., Kong, S., Zhang, Y., et al. 2016, ApJL, 821, L3

Tanaka, K. E. I., Tan, J. C., \& Zhang, Y. 2017, ApJ, 835, 32

van Dishoeck, E. F., Blake, G. A., Draine, B. T., \& Lunine, J. I. 1993, in Protostars and Planets III, ed. E. H. Levy \& J. I. Lunine (Tucson, AZ: Univ. Arizona Press), 163

Wang, K., Zhang, Q., Testi, L., et al. 2014, MNRAS, 439, 3275

Wang, K., Zhang, Q., Wu, Y., Li, H.-b., \& Zhang, H. 2012, ApJL, 745, L30

Wang, P., Li, Z.-Y., Abel, T., \& Nakamura, F. 2010, ApJ, 709, 27

Wilson, T. L. 1999, RPPh, 62, 143

Zhang, Y., \& Tan, J. C. 2015, ApJL, 802, L15

Zhang, Y., Tan, J. C., \& Hosokawa, T. 2014, ApJ, 788, 166 